\title{
छs \\ Achromatic telescopic squeezing scheme and application to the LHC and its luminosity upgrade
}

\author{
Stéphane Fartoukh* \\ CERN, CH 1211 Geneva 23, Switzerland \\ (Received 26 July 2013; published 19 November 2013)
}

\begin{abstract}
A novel optics concept, the achromatic telescopic squeezing (ATS) scheme has been invented in the context of the Large Hadron Collider (LHC) upgrade studies, and chosen as the baseline scheme for the optics and layout of the recently approved high luminosity LHC project (HL-LHC). This scheme offers an extremely powerful and flexible machinery in order to strongly reduce $\beta^{*}$ in a symmetric or asymmetric way (i.e. without necessarily imposing the same $\beta^{*}$ in both planes), while perfectly controlling the chromatic aberrations induced, namely the linear and nonlinear chromaticities, the off-momentum $\beta$ beating, and the spurious dispersion from the large crossing angle which is required at small $\beta^{*}$ in the particular case of the (HL)-LHC. The initial motivations of the scheme will be reviewed, followed by a detailed description of its fundamental theoretical foundations. An effective construction of ATS optics will be given and its main features illustrated in the case of the LHC and HL-LHC.
\end{abstract}

DOI: $10.1103 /$ PhysRevSTAB.16.111002

PACS numbers: 29.27.- a, 41.85.-p

\section{INTRODUCTION AND MOTIVATIONS}

\section{A. The general context}

Reducing the beam spot size at the interaction point (IP), which is acting on $\beta^{*}$ at constant transverse emittances, is a key ingredient to boost the performance of any collider. However, a strong reduction of $\beta^{*}$ requires in principle longer final focus systems (FFS) in order to enable a smooth matching of the optics between the IP and the regular lattice of the machine, which are the arcs in the case of a circular machine, or the two facing linacs in the case of a linear collider. For a machine which is still in its design phase, this modification is in general feasible, but a priori far from being neutral in terms of cost and planning. On the other hand, appropriate solutions, or even acceptable compromises, might be very hard if not impossible to find, when strongly relying on this approach for upgrading the performance of a machine which is already built.

Another prerequisite to reduce $\beta^{*}$ might also be a reduction of $L^{*}$, which is the distance between the last final focus quadrupole and the interaction point. This intervention aims at mitigating the inevitable increase of the chromatic aberrations due to the larger $\beta$ functions in the final focus quadrupoles induced at smaller $\beta^{*}$ : the momentum $\beta^{*}$ acceptance in the specific case of a linear collider, or, in the case of a circular machine, the linear and nonlinear chromaticities, the off-momentum $\beta$ beating, and the spurious dispersion induced by an eventual nonzero crossing

\footnotetext{
*stephane.fartoukh@cern.ch
}

Published by the American Physical Society under the terms of the Creative Commons Attribution 3.0 License. Further distribution of this work must maintain attribution to the author(s) and the published article's title, journal citation, and DOI. angle imposed at the IP. Sharper modifications might also consist in a complete redesign of the chromatic correction system initially foreseen, making it stronger and more efficient. However, the price to pay might be again in this case a substantial lengthening of the final focus systems of the collider or, more specifically for circular machines, a delicate surgery into the heart of the lattice by replacing the existing chromaticity sextupoles by new ones, much stronger, in all the arcs of the ring.

The situation is therefore very complicated for a circular collider which is already built, with hard constraints imposed on $L^{*}$ by the size of the existing experiment detectors, and strictly no flexibility for a possible extension of the low- $\beta$ insertions (IRs) which are obviously defined by the geometry of the existing tunnel. The so-called achromatic telescopic squeezing (ATS) scheme is a novel optics concept which offers a complete solution to the above issues. After a detailed introduction given in the next section to discuss the motivations of this work in the case of the Large Hadron Collider (LHC) [1], the fundamental principles of the ATS scheme will be presented in detail in Sec. II, then illustrated in Sec. III in the framework of the luminosity upgrade program of the LHC (HL-LHC [2]).

\section{B. The LHC with its optics limitations at low $\beta^{*}$ \\ 1. A rapid description of the $\mathrm{LHC}$ ring}

As many circular machines, the LHC consists of a certain number of arcs, forming a periodic lattice which is interrupted by service and experimental low- $\beta$ insertions; namely, the momentum and betatron cleaning insertions IR3 and IR7, the rf insertion in IR4 containing as well most of the beam instrumentation, the dump insertion in IR6, the low-luminosity insertions IR2 and IR8 housing the Alice and $\mathrm{LHCb}$ experiments (and also used for the beam 


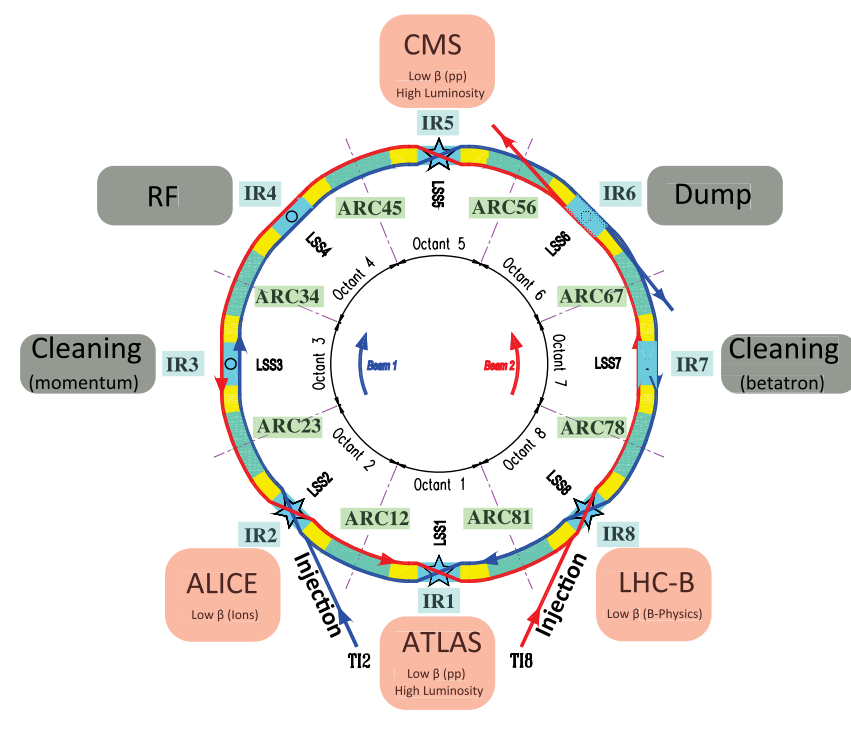

FIG. 1. Schematic layout of the LHC [1].

injection), and finally the two high luminosity insertions IR1 and IR5 for the ATLAS and CMS experiments (see Fig. 1).

\section{The LHC experimental insertions in brief}

The two LHC beams circulate in separated apertures in most of the ring, except in the experimental insertions where they share the same vacuum chamber between the IP and a so-called Y-chamber located in between the separation and recombination dipoles D1 and D2 (see Fig. 2). The main field of the final focus quadrupoles therefore impacts in an opposite way on the two counterrotating LHC proton beams. Aiming at simultaneous optics solutions for both beams led to the design of an optics which is antisymmetric between the left and the right sides of the IP for a given beam, and between Beam1 and Beam2 on a given side of the IP, and to a layout solution with a final triplet rather than with a final doublet.
The four experimental insertions of the LHC are based on the same conceptual layout (see Fig. 2). From the optics point of view, these IRs contain three main parts: (i) a region which is shared by the two beams from the interaction point (IP) to the separation dipole D1, with the inner triplet in between installed at $L^{*}=23 \mathrm{~m}$; (ii) the matching section, starting at the recombination dipole D2 and containing three individually powered two-in-one quadrupole magnets Q4, Q5, and Q6; (iii) the dispersion suppressor starting with Q7 followed by the first arc dipole, and containing four individually powered two-in-one aperture quadrupole magnets (Q7 to Q10). Strictly speaking, the dispersion suppressor extends up to Q13, with the arc quadrupoles Q11, Q12, and Q13 equipped with three individually powered trim quadrupoles.

The linear optics becomes strictly periodic as of Q13, where the arc quadrupoles are arranged into 21 regular FODO cells (i.e. focusing quadrupole, drift space, defocusing quadrupole, drift space). In practice, as for most of the modern colliders, matching the optics of an LHC low- $\beta$ insertion consists in satisfying a certain number of constraints including $\beta^{*}$, but not only, and using the quadrupoles of the matching section and dispersion suppressor, while the gradient of the inner triplet is kept quasiconstant. Starting from the periodic optical functions of the arcs on the left side of the insertion (Q13.L), 14 optics constraints shall be fulfilled; namely, the horizontal and vertical betatron phase advances from Q13.L to Q13.R, which are kept constant for any $\beta^{*}$ for preserving the betatron tunes during the optics squeeze, and the Twiss $\left(\beta_{x, y}, \alpha_{x, y}\right)$ and dispersion $\left(D_{x}, D_{x}^{\prime}\right)$ parameters, both at the IP and at the exit of the insertion (Q13.R), where these parameters shall again coincide with the periodic conditions of the next arc.

The ATS scheme will complement this concept with the so-called presqueezed optics which relies on additional phase matching conditions to be met on several sections of the ring. Then it will completely modify the standard

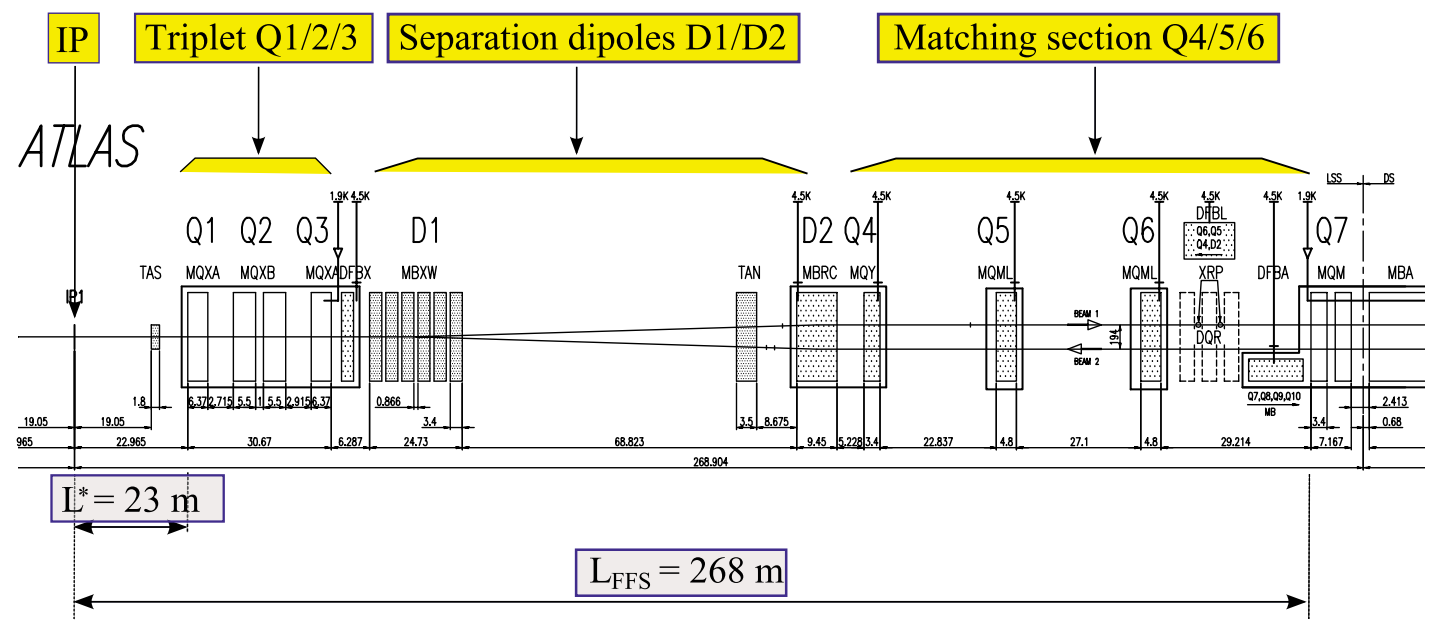

FIG. 2. Low- $\beta$ insertion of the LHC from the interaction point to the entry of the dispersion suppressor [1]. 

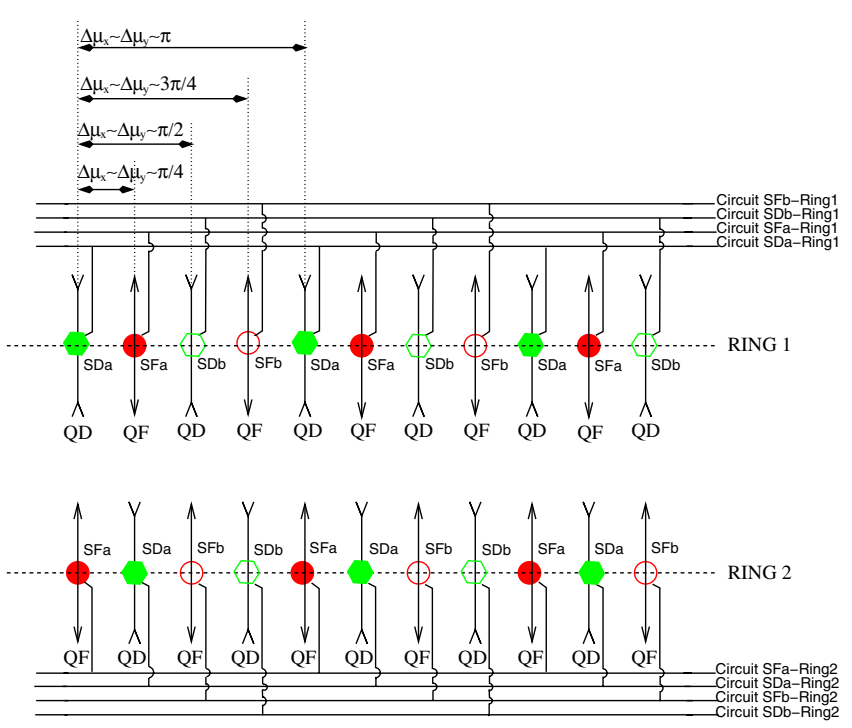

FIG. 3. Schematic layout of the LHC arc sextupole powering scheme [3]. For each beam, and in each arc of the machine, the focusing and defocusing sextupoles are split into two interleaved families. For the nominal LHC optics, the horizontal and vertical betatron phase advances are close to $\pi$ between two consecutive sextupoles belonging to the same family. These phases are matched to exactly $\pi$ for ATS optics.

approach introducing a second (telescopic) part for the squeeze, in order to further reduce $\beta^{*}$ in a somehow achromatic way (see Sec. II).

\section{The LHC chromatic correction system in brief}

The chromatic correction system of the LHC is hosted in the arcs, with two interleaved families of sextupoles, in each of the two transverse planes and each of the eight sectors of the ring (see Fig. 3). This makes a total of 32 independent sextupole families per beam for the entire ring. The nominal LHC optics is matched with betatron phase advances close, but not exactly equal to 90 degrees in the arc cells. This induces a phase split of about $\Delta \mu_{x}-$ $\Delta \mu_{y} \sim \pi$ in each sector of the ring, contributing to about $80 \%$ of the overall integer tune split of $5\left(Q_{x, y}=\right.$ $64.31 / 59.32$ in collision). This strategy was implemented in the early design of the machine (see e.g. [4] and references therein), and kept unchanged since then. The aim was indeed to mitigate strong nonlinear resonance driving terms which could have been driven by large systematic field imperfections in the LHC main dipoles. A posteriori, this optics choice is no longer justified, with a field quality much better than initially anticipated in the main magnets. De facto this would allow one to go back to phase advances much closer, if not strictly equal to $\pi / 2$ in the LHC arc cells. On the contrary, as will be demonstrated later, preserving such an optics would prevent one to use efficiently the flexibility offered by the existing LHC sextupole powering scheme in order to control properly the chromatic properties of the collision optics at very small $\beta^{*}$. The chromatic observables that become critical at low $\beta^{*}$ are indeed not only the linear chromaticity, but also other aberrations, extremely sensitive to the phasing properties of the linear optics, such as the nonlinear chromaticity, the off-momentum $\beta$ beating $\partial \beta / \partial \delta$, and, in the case of the LHC, the horizontal or vertical spurious dispersion driven by the crossing scheme (crossing angle and parallel separation) which are implemented in the four experimental insertions of the ring.

\section{The challenge of reducing $\beta^{*}$ in the $\mathrm{LHC}$}

In this context, reducing $\beta^{*}$ with respect to its nominal value of $55 \mathrm{~cm}$ [1] leads rapidly to a series of limitations. These limitations are driven on one side by the mechanical aperture available in the final focus quadrupoles, that is the inner triplet (IT), but also coming from the rest of the ring. Concerning the IT aperture-related constraints, it was shown that long and weak enough quadrupoles (basically with constant integrated gradient) can always offer more aperture than actually needed by the beam, regardless of $\beta^{*}$ and of the technology chosen for the triplet $[5,6]$. Indeed, by decreasing the triplet gradient $G$ at constant $\beta^{*}$ (but of course making it longer to preserve its integrated strength), the IT aperture can in principle be increased with $1 / G$ at constant peak field. Under these conditions, the aperture needed for the beam increases much less rapidly with a rough scaling like $1 / G^{1 / 4}$, or, said differently, the peak $\beta$ function reached in the triplet is found to increase with $1 / \sqrt{G}$ :

$$
\hat{\beta}_{\mathrm{IT}} \propto \frac{1}{\beta^{*} \sqrt{G}} .
$$

Therefore, the real optics challenge for low $\beta^{*}$ is elsewhere, on the "nontriplet" side of the machine, where a series of limitations were clearly identified and classified in the framework of the former upgrade project of the LHC [7]. While of very different nature, all these limitations can be quantified by the maximum possible peak $\beta$ function which is permitted in the inner triplet, namely,

$$
\hat{\beta}_{\text {IT }}<\beta_{\max }
$$

where $\beta_{\max }$ shall be understood as a limit given by the ring and not by the final focus quadrupoles. Indeed, the peak $\beta$ function reached in the triplet shall then be matched to the regular optics of the arcs within the fixed distance given by the length the low- $\beta$ insertions, and within the aperture and gradient limits of the IR magnets. Finally, a clear strategy shall be established for the correction of the chromatic aberrations induced, and ideally within the available strength of the existing chromaticity sextupoles. Assuming an upgrade of the LHC which would only consist of replacing the existing inner triplet, and no deep conceptual 
changes in general beam optics for circular colliders, these limitations can rapidly turn into hard limits driven by the existing hardware all around the ring; namely, the mechanical acceptance of the existing low- $\beta$ insertions (not only the triplet but also most of the magnets of the matching section), the gradient limits of the matching quadrupoles (in particular Q6 and Q7 pushed to very low and high gradients, respectively), and the strength limits of the arc sextupoles.

Taking these considerations into account, the maximum possible $\beta_{\max }$ permitted in any new LHC triplet was found to be around $11 \mathrm{~km}$ [7], compared to $4.5 \mathrm{~km}$ for the nominal collision optics of the LHC. This defined at that time an optimal aperture of $120 \mathrm{~mm}$ for the next triplet generation, regardless of the technology chosen. The corresponding minimum possible $\beta^{*}$ was then found to be $\beta_{\min }^{*}=30 \mathrm{~cm}$ for the Nb-Ti technology $(120 \mathrm{~T} / \mathrm{m}-120 \mathrm{~mm}$ triplet) and, according to the scaling law given in (1), only $\sim 20 \%$ less, that is $\beta_{\min }^{*}=24-25 \mathrm{~cm}$, for the $\mathrm{Nb}_{3} \mathrm{Sn}$ technology a priori compatible with a $50 \%$ higher gradient $(180 \mathrm{~T} / \mathrm{m}-120 \mathrm{~mm}$ triplet).

The ATS scheme which was invented and developed afterward (see [8] for the first description of the basic concepts) offers the possibility to go well beyond, and therefore completely modified the above conclusions, while offering a solid ground to the HL-LHC target of $\beta^{*}=10-15 \mathrm{~cm}$ [2]. The basic concepts and technical implementation of the ATS scheme are described in detail in the next section taking the (HL)-LHC as a unique example. It is however worth mentioning a certain effort presently on-going in order to apply or adapt the ATS concept to other existing or future machines, such as the $\mathrm{LHeC}$ [9] or (e)RHIC [10].

\section{THE THEORETICAL FOUNDATIONS OF THE ATS}

\section{A. The basic principles}

Concerning the first optics limitation mentioned previously (aperture limitation), the only solution is to equip the machine with new magnets of larger aperture whenever needed. For the HL-LHC, this means that a few magnets shall be replaced in the matching section of IR1 and IR5, including of course new inner triplets. Concerning the poor optics flexibility observed at low $\beta^{*}$, one possibility is to envisage floating matching conditions at the boundaries of the high luminosity insertions. The idea is to maintain the dispersion matching constraints at the entry and exit of the low- $\beta$ insertions, but to allow the neighboring insertions on either side (IR8/2 for IR1 and IR4/6 for IR5) to contribute as well to the squeeze, at least below a certain $\beta^{*}$. This $\beta^{*}$ will be called later the presqueezed $\beta^{*}$. As a result, $\beta$-beating waves are generated in the arcs which are directly adjacent to the low- $\beta$ insertions, namely the $\operatorname{arcs} 45$, 56,81 , and 12 (see the example of arc 45 illustrated in Fig. 4). Assuming a phase advance per arc cell strictly matched to $\pi / 2$ in these arcs, and if correctly phased with respect to the IP, these waves will reach their maximum at every other sextupole, i.e. at the sextupoles belonging to the same electrical circuit (see Fig. 3). Consequently, the chromatic correction efficiency of these sextupoles will drastically increase at constant strength, which offers a definite cure for the third and last optics limitation mentioned at the end of the previous section.

Implementing the ATS concept means in practice to split into two parts the transition between the injection and collision optics. First a presqueeze of $\beta^{*}$ is achieved following the standard IR matching procedure described in Sec. IB 2, but with additional matching constraints imposed individually for the left and right phase advances of the high luminosity insertions (see Sec. II B 1 for the motivation). The presqueeze is pushed until reaching certain limits, either related to the gradient of the matching quadrupoles or to the strength of the lattice sextupoles. The chromatic limit is generally the first being reached both for the LHC, and most of the new triplet layouts studied so far for the HL-LHC. For a further reduction of $\beta^{*}$, the presqueeze is followed by the telescopic squeeze, acting only on the matching quadrupoles equipping the neighboring insertions that are on either side of each high luminosity insertion (i.e. IR8 and IR2 for squeezing IR1, and IR4 and IR6 for squeezing IR5). The telescopic squeeze is therefore operated at strictly constant settings for the quadrupoles equipping the high luminosity insertions proper, but also at quasiconstant strength for the lattice sextupoles (see Sec. II B 2).

This novel approach is particularly well suited to the LHC for the two following reasons. First, due to the large dynamic range of the machine in energy, from $450 \mathrm{GeV}$ to $7 \mathrm{TeV}$, and the reduction in proportion of the transverse emittances during the ramp, the peak $\beta$ functions in the arcs could in principle be increased by a factor of 16 without exceeding any aperture-related limits at flattop energy. Second, the quadrupole magnets of the supporting insertions IR8, IR2, IR4, and IR6 are either moderately pushed, which is the case for the experimental insertions IR8 and IR2 (assuming a $\beta^{*}$ of a few meters in protonproton collision mode), or not pushed at all in the case of IR4 and IR6 for which the injection optics is kept unchanged during the whole cycle of the machine. Therefore most of the ingredients are in fact already available in the existing LHC to blow up the $\beta$ functions in the arcs $81 / 12 / 45 / 56$ at $7 \mathrm{TeV}$ and implement the principles of the ATS scheme.

\section{B. Chromatic correction}

The technical implementation of the ATS scheme imposes in a first step a detailed analysis of the chromatic aberrations induced by the final focus quadrupoles at low $\beta^{*}$, and of the means that shall be implemented in order to properly correct them. These chromatic aberrations are driven by the following quantities: 


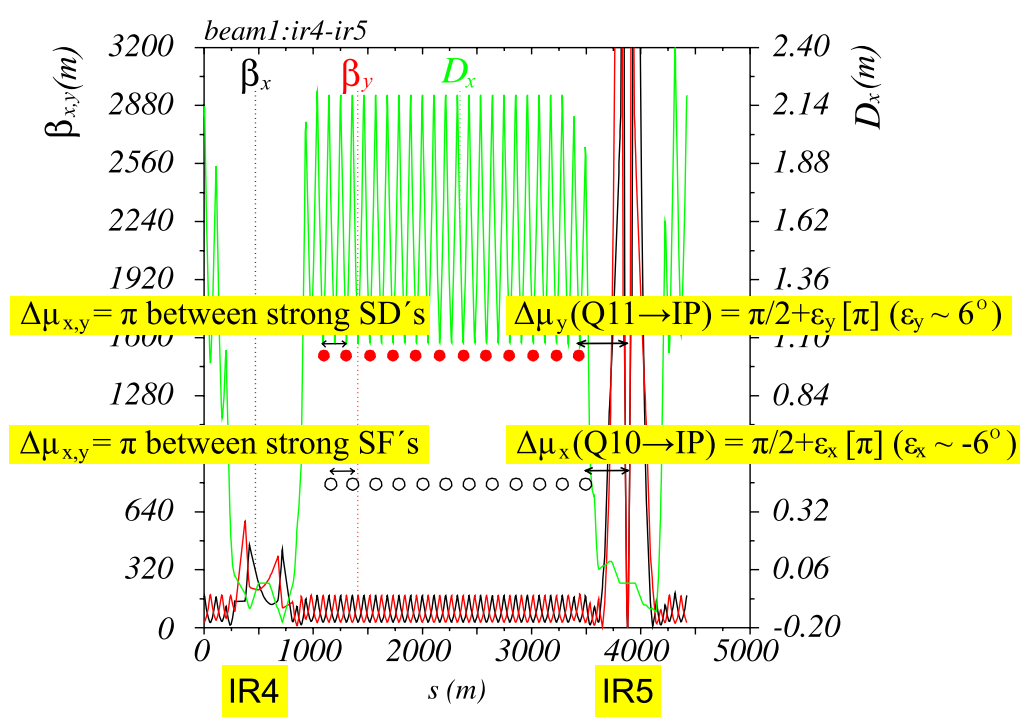

(a): Matched LHC arc optics

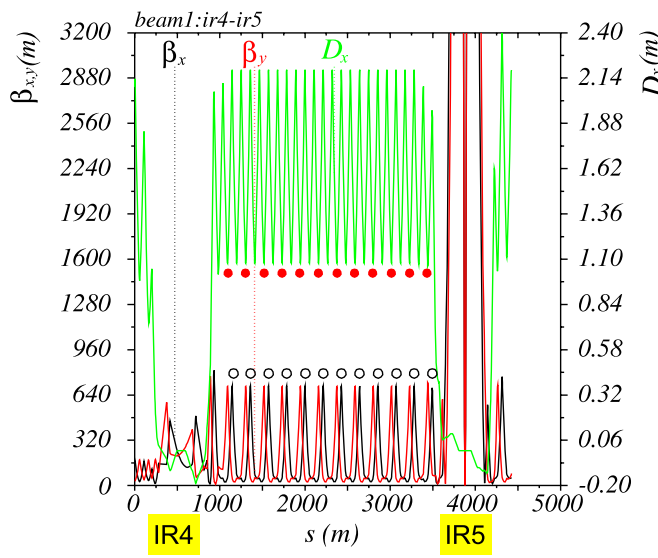

(b): Mismatched for round telescopic optics

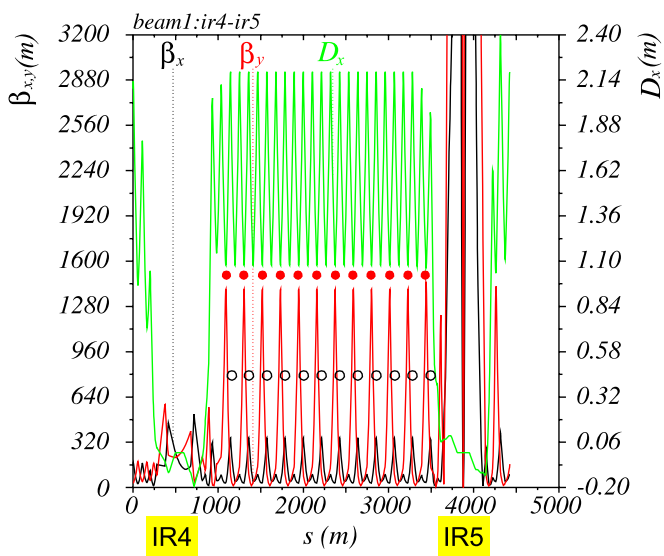

(c): Mismatched for flat telescopic optics

FIG. 4. Modification of the $\beta$ functions in the arc 45 of the LHC during the telescopic squeeze, starting from a round presqueezed optics with $\beta_{0}^{*}=40 \mathrm{~cm}$ at IP5 and specific phasing properties (a), and reaching two possible collision optics, either round with $\tilde{\beta}_{x, y}^{*}=10 \mathrm{~cm}$ (b) or flat with $\tilde{\beta}_{x}^{*}=20 \mathrm{~cm}$ and $\tilde{\beta}_{y}^{*}=5 \mathrm{~cm}(\mathrm{c})$. The telescopic squeeze is achieved by acting only on the matching quadrupoles of IR4 on the left (and IR6 on the right), as soon as specific betatron phase advances have been achieved for the presqueezed optics (see Sec. II B 1). The peak $\beta$ functions in the arcs are increasing in proportion to the reduction of $\beta^{*}$, i.e. by a factor of 4 in both planes for case (b), and by the factors 2 and 8 in the horizontal and vertical planes, respectively, for case (c). The empty and full markers indicate the location of the focusing and defocusing sextupoles, respectively, which participate to the chromatic correction of the LHC inner triplet. These sextupoles can be operated at quasiconstant settings during the telescopic squeeze with the $\beta$ functions increasing proportionally with $\beta_{0}^{*} / \tilde{\beta}^{*}$ at their respective location (see Sec. II B 2).

$$
I_{x, y}^{L, R} \stackrel{\text { def }}{=} \pm \int_{\text {triplet }(L, R)} d s K_{1}(s) \beta_{x, y}(s)
$$

where the integrals are taken over the final focus quadrupoles equipping the left $(\mathrm{L})$ or right $(\mathrm{R})$ side of a given high luminosity insertion, and $K_{1}(s)$ denotes their normalized gradient (with the convention $K_{1}<0$ for an horizontally focusing quadrupole). Within a factor $4 \pi$, the above quantities simply represent the main contribution of the low- $\beta$ insertions to the natural chromaticity of the machine when the optics is squeezed. These integrals are therefore negative, with a very accurate scaling at low $\beta^{*}$ given by

$$
I_{x, y}^{L, R} \propto 1 / \beta_{x, y}^{*},
$$

both for a standard squeeze and the two-stage procedure of the ATS. For a purely symmetric optics with respect to the IP, the left and right integrals are identical in each of the 
two transverse planes. In the specific case of the LHC, the triplet powering is actually antisymmetric with respect to the IP, leading to the following relation:

$$
\beta_{x}^{*} I_{x}^{L}=\beta_{y}^{*} I_{y}^{R} \quad \text { and } \quad \beta_{x}^{*} I_{x}^{R}=\beta_{y}^{*} I_{y}^{L} .
$$

In particular, even for round optics $\left(\beta_{x}^{*}=\beta_{y}^{*}\right)$, the left and right integrals $I_{x, y}^{L, R}$ are not necessarily the same in a given plane. For the existing LHC triplet operating at $205 \mathrm{~T} / \mathrm{m}$, the two integrals differ by about $\Delta I /(2 I) \sim 10 \%$ (see e.g. [3]). This difference tends however to decrease for triplets operating at lower gradient. It is therefore relevant to introduce the following average quantities:

$$
I_{x, y}=\frac{1}{2}\left(I_{x, y}^{L}+I_{x, y}^{R}\right) \quad \text { and } \quad \Delta I_{x, y}= \pm \frac{1}{2}\left(I_{x, y}^{L}-I_{x, y}^{R}\right),
$$

with

$$
I_{x}=\frac{\beta_{y}^{*}}{\beta_{x}^{*}} I_{y} \quad \text { and } \quad \Delta I_{x}=\frac{\beta_{y}^{*}}{\beta_{x}^{*}} \Delta I_{y},
$$

which hold both for a left/right symmetric or antisymmetric powering configuration for the final focus quadrupoles.

A proper chromatic correction consists of course in compensating the contribution of the inner triplet to the linear chromaticity $Q_{x, y}^{\prime}$,

$$
Q_{x, y}^{\prime} \propto \int_{0}^{C} d s\left[K_{1}(s)-K_{2}(s) D_{x}(s)\right] \beta_{x, y}(s) \equiv 0,
$$

but also in controlling the chromatic variations of the Twiss parameters, which are directly connected to the $(2,0)$ and $(0,2)$ chromatic driving terms:

$$
\begin{aligned}
& {\left[\frac{\partial_{\delta} \beta_{x, y}}{\beta_{x, y}}-j\left(\partial_{\delta} \alpha_{x, y}-\alpha_{x, y} \frac{\partial_{\delta} \beta_{x, y}}{\beta_{x, y}}\right)\right]_{s=s_{0}}} \\
& \quad \propto c_{(2,0),(0,2)}\left(s_{0}\right) \\
& \quad \stackrel{\operatorname{def}}{=} \int_{s_{0}}^{s_{0}+C} d s\left[K_{1}(s)-K_{2}(s) D_{x}(s)\right] \beta_{x, y}(s) e^{2 j\left[\mu_{x, y}(s)-\mu_{x, y}\left(s_{0}\right)\right]} \\
& \quad \equiv 0
\end{aligned}
$$

where $C$ is the ring circumference and the quantities $K_{1,2}$ represent the normalized strengths of the quadrupoles and sextupoles of the lattice, respectively. Taking the modulus of the complex functions occurring in the left-hand side (lhs) of Eq. (9) leads to the well-known chromatic Montague functions $W_{x, y}$, although the direct connection with the $(2,0)$ and $(0,2)$ resonance driving terms $c_{(2,0),(0,2)}$ is generally less familiar.
Fulfilling the condition (9) at specific locations in the ring (inner triplets, collimation insertion) becomes absolutely necessary when reducing $\beta^{*}$, with otherwise strong nonlinear chromatic variations of the betatron tunes which reduce the momentum acceptance of the linear optics, and a large off-momentum $\beta$ beating all around the ring, possibly impacting on the collimation hierarchy and severely on the mechanical acceptance of the inner triplet [see e.g. Figs. 6(b), 7(b), and 7(d)]. On the other hand, the condition (9) assumes that specific phasing conditions are met between the source, i.e. the final focus quadrupoles, and the various correctors involved, i.e. the arcs where the sextupoles are located, which is not the case for the nominal LHC collision optics. A dedicated optics rematching of the entire ring is therefore required as a fundamental prerequisite for the implementation of the ATS scheme.

\section{The proper phasing of the presqueezed optics and its chromatic correction}

In this section the $\beta$ functions are assumed to be matched in the $\operatorname{arcs}\left(\beta^{*} \geq \beta_{\text {presqueeze }}^{*}\right)$. The goal is to achieve the chromatic correction of each inner triplet by using one single arc of sextupoles on either side of the IP [e.g. the arc 45 for the chromatic correction of the triplet on the left side of IP5, as shown in Fig. 4(a)]. Assuming a phase advance in the arc cell close to 90 degrees, two consecutive focusing or defocusing sextupoles, $\mathrm{SFa} / \mathrm{b}$ or $\mathrm{SDa} / \mathrm{b}$ (see Fig. 3) clearly act against each other for the excitation of the $(2,0)$ or $(0,2)$ driving terms [see the phasor $e^{2 j \mu(s)}$ occurring in the integrand of Eq. (9)]. Therefore, in the arcs directly adjacent to the low- $\beta$ insertions, only one sextupole family per plane, e.g. the families SFa and SDa, shall be used for the chromatic correction of the inner triplet. In the following these families will be referred as the "strong" sextupole families, by opposition to the "weak" sextupole families for the other two SF and SD families in a given arc, and the labels "a" or " $b$ " will be omitted. For a similar reason, since the off-momentum $\beta$ beating generated by each strong sextupole goes with twice the betatron phases, the coherence of wave, and therefore the efficiency of the correction, will be maximized if the phase advances in the arc cell are matched to exactly $\pi / 2$ in both planes. This condition is equivalent to a phase advance of $\pi$ in between two consecutive strong sextupoles of the same family, and to $\pi / 4$, or $3 \pi / 4$, between two consecutive strong sextupoles SF and SD (see Fig. 3):

$$
\Delta \mu_{x, y}^{\text {cell }} \equiv \frac{\pi}{2} \Rightarrow\left\{\begin{array}{l}
\Delta \mu_{x, y}^{S F^{(n)} \rightarrow S F^{(n+1)}}=\Delta \mu_{x, y}^{S D^{(n)} \rightarrow S D^{(n+1)}}=\pi \\
\Delta \mu_{x, y}^{S F^{(n)} \rightarrow S D^{(n)}}=\frac{\pi}{4} \quad\left(\text { or } \frac{3 \pi}{4}\right) \quad \text { and } \quad \Delta \mu_{x, y}^{S D^{(n)} \rightarrow S F^{(n+1)}}=\frac{3 \pi}{4} \quad\left(\text { or } \frac{\pi}{4}\right) .
\end{array}\right.
$$

Under these conditions, and averaging the chromatic correction between the left and the right sides of a given low- $\beta$ insertion (in order to balance the sextupole settings on either side of the IP), the conditions (8) and (9) simply read

$$
N_{F}^{L, R} \beta_{x_{F}} D_{x_{F}} \Delta K_{2_{F}}^{L, R}+N_{D}^{L, R} \beta_{x_{D}} D_{x_{D}} \Delta K_{2_{D}}^{L, R} \equiv I_{x}, \quad N_{F}^{L, R} \beta_{y_{F}} D_{x_{F}} \Delta K_{2_{F}}^{L, R}+N_{D}^{L, R} \beta_{y_{D}} D_{x_{D}} \Delta K_{2_{D}}^{L, R} \equiv-I_{y},
$$


and

$$
\begin{aligned}
e^{2 j\left(\Delta \mu_{x}^{L, R}-\pi / 2\right)}\left[N_{F}^{L, R} \beta_{x_{F}} D_{x_{F}} \Delta K_{2_{F}}^{L, R}+e^{2 j \Delta \mu_{x_{F}}^{L, R}} N_{D}^{L, R} \beta_{x_{D}} D_{x_{D}} \Delta K_{2_{D}}^{L, R}\right] & \equiv I_{x}, \\
e^{2 j\left(\Delta \mu_{y}^{L, R}-\pi / 2\right)}\left[e^{-2 j \Delta \mu_{y_{F D}}^{L, R}} N_{F}^{L, R} \beta_{y_{F}} D_{x_{F}} \Delta K_{2_{F}}^{L, R}+N_{D}^{L, R} \beta_{y_{D}} D_{x_{D}} \Delta K_{2_{D}}^{L, R}\right] & \equiv-I_{y},
\end{aligned}
$$

where the chromatic quantities $I_{x, y}$ are defined in Eq. (6), and the following notations and approximations have been used. $N_{F}^{L, R}$ (respectively $N_{D}^{L, R}$ ) denotes the number of strong sextupoles SF (respectively $\mathrm{SD}$ ) which are located in the arc on the left (L) or right (R) side of the IP, and $\Delta K_{2_{F, D}, R}$ represents their change of normalized strength with respect to the injection optics. The quantities $\beta_{x_{F, D}}, \beta_{y_{F, D}}$, and $D_{x_{F, D}}$ are the horizontal and vertical $\beta$ functions, and the horizontal dispersion, respectively, which are reached at the strong sextupoles SF or SD. Since the phase advance per arc cell is assumed to be matched to $\pi / 2$ in both planes, we have in particular

$$
\beta_{x_{F}}=\beta_{y_{D}} \stackrel{\text { def }}{=} \beta_{F}, \quad \beta_{y_{F}}=\beta_{x_{D}} \stackrel{\text { def }}{=} \beta_{D},
$$

with $\beta_{F / D} \approx 175 / 35 \mathrm{~m}$ in the LHC. The phases $\Delta \mu_{x}^{L, R}$ (respectively $\Delta \mu_{y}^{L, R}$ ) represent the left and right phase advances of the low- $\beta$ insertions, more precisely, the horizontal (respectively vertical) betatron phase advances between the IP and the first strong focusing (respectively defocusing) sextupole installed on the left or right of the IP. The phases $\Delta \mu_{x_{F D}}^{L, R}$ (respectively $\Delta \mu_{y_{F D}}^{L, R}$ ) denote the horizontal (respectively vertical) betatron phase advance from the last focusing to the last defocusing strong sextupole in the arc on the left or right side of the IP. According to the condition (10), this phase can be either $\pi / 4$ or $3 \pi / 4$ in both planes, depending on the choice of the strong sextupole families amongst the families (a) or (b):

$$
e^{ \pm 2 j \Delta \mu_{x_{F D}}^{L, R}}=e^{ \pm 2 j \Delta \mu_{y_{F D}}^{L, R}}= \pm j \xi^{L, R} \text { with } \xi^{L, R}= \pm 1,
$$

which does not need to be further specified. Finally, the above relations assume that the betatron phase advances are quasiconstant over the inner triplet quadrupoles, then jump by $\pi / 2$ at the IP. This is in general a very good approximation for $\beta^{*} \lesssim 1 \mathrm{~m}$.

On each side of the IP, the relations (11) and (12) can be seen as a set of six real conditions. The two conditions forming Eq. (11) are related to the correction of the linear chromaticity $Q^{\prime}$. Since the LHC optics is left/right antisymmetric, these relations taken individually on the left or right side of the low- $\beta$ insertion tend to slightly overcompensate or undercompensate the contribution of the final focus quadrupoles to $Q^{\prime}$, by the amount $\pm \Delta I_{x, y} /(4 \pi)$ [see Eq. (6)]. On the other hand, fulfilling these relations on both sides of the IP guarantees a full chromaticity correction of the insertion, using only two arcs of sextupoles. The two conditions in Eq. (12) are related to the compensation of the $(2,0)$ and $(0,2)$ complex resonance driving terms, and therefore form a set of four real conditions. The lhs of these two conditions basically describes the amplitude and phase of the off-momentum $\beta$-beating wave induced by the strong sextupoles. This wave shall arrive out of phase by $\pi / 2$ at the location of the inner triplet for a proper compensation of the chromatic betatron kick which is described by the real quantity $I_{x, y}$. Assuming these conditions to be met, the off-momentum $\beta$ beating $\partial_{\delta} \beta$ will vanish both in the final focus quadrupoles and at the IP. On the other hand, the off-momentum $\alpha$ beating will be slightly overcompensated (or undercompensated) at these two locations (still due to the antisymmetric LHC optics), but perfectly compensated considering globally the full insertion and its two adjacent sectors.

Only four parameters are available per IP side for the chromatic correction, namely the settings $\Delta K_{2_{F, D}^{L, R}}$ of the strong sextupoles, and the left (or right) IR phase advances $\Delta \mu_{x, y}^{L, R}$. These four parameters are determined by solving the second condition (12). After some algebra and using (7), one gets

$$
\begin{aligned}
& \Delta K_{2_{F}}^{L, R}=\frac{1}{N_{F}^{L, R} \beta_{F} D_{x_{F}}} \frac{\sqrt{1-r_{F D}^{2}\left(\frac{\left.\beta_{x}^{*}\right)_{y}^{2}}{\beta_{y}^{*}}\right.}}{\sqrt{1-r_{F D}^{4}}} \times I_{x}, \\
& \Delta K_{2_{D}}^{L, R}=-\frac{1}{N_{D}^{L, R} \beta_{F} D_{x_{D}}} \frac{\sqrt{1-r_{F D}^{2}\left(\frac{\beta_{y}^{*} \beta_{x}^{*}}{\beta^{2}}\right.}}{\sqrt{1-r_{F D}^{4}}} \times I_{y},
\end{aligned}
$$

and

$$
\begin{aligned}
& \exp \left[2 j \Delta \mu_{x}^{L, R}\right]=-\frac{\sqrt{1-r_{F D}^{2}\left(\frac{\left.\beta_{x}^{*}\right)_{y}^{2}}{\beta^{*}}\right.}}{\sqrt{1-r_{F D}^{4}}} \\
& -j \xi^{L, R} r_{F D} \frac{\beta_{x}^{*}}{\beta_{y}^{*}} \frac{\sqrt{1-r_{F D}^{2}\left(\frac{\beta_{y}^{*}}{\beta_{x}^{*}}\right)^{2}}}{\sqrt{1-r_{F D}^{4}}}, \\
& \exp \left[2 j \Delta \mu_{y}^{L, R}\right]=-\frac{\sqrt{1-r_{F D}^{2}\left(\frac{\beta_{y}^{*}}{\beta_{x}^{*}}\right)^{2}}}{\sqrt{1-r_{F D}^{4}}} \\
& +j \xi^{L, R} r_{F D} \frac{\beta_{y}^{*}}{\beta_{x}^{*}} \frac{\sqrt{1-r_{F D}^{2}\left(\frac{\left.\beta_{x}^{*}\right)^{2}}{\beta_{y}^{*}}\right.}}{\sqrt{1-r_{F D}^{4}}},
\end{aligned}
$$

where the quantity $r_{F D}$ represents the aspect ratio of the $\beta$ functions at the strong sextupoles:

$$
r_{F D} \stackrel{\text { def }}{=} \frac{\beta_{D}}{\beta_{F}} \approx \frac{1-\sin \left[\Delta \mu^{\text {cell }} / 2\right]}{1+\sin \left[\Delta \mu^{\text {cell }} / 2\right]}=0.1715 \ldots,
$$


for a standard $\pi / 2$ FODO structure where the lattice sextupoles are installed close enough to the main quadrupoles.

Therefore, if the presqueeze is achieved at constant $\beta^{*}$ aspect ratio, not only the overall IR phase shall be kept constant in order to work at constant tune, but also the left and right phase advances $\Delta \mu_{x, y}^{L, R}$. The latter are defined in Eq. (16) within a integer multiple of $\pi$, as soon as the strong sextupole families have been chosen amongst the available families (a) and (b), which, in practice, will be driven by the limited flexibility in phase of the high luminosity insertions at low $\beta^{*}$. The existence of the solutions (15) and (16) depends however on the $\beta^{*}$ aspect ratio, which shall be larger than the aspect ratio $r_{F D}$ :

$$
r_{F D} \leq \min \left(\frac{\beta_{x}^{*}}{\beta_{y}^{*}}, \frac{\beta_{y}^{*}}{\beta_{x}^{*}}\right) .
$$

For the sake of simplification however, but without losing too much in generality, we will assume in the following that the presqueezed optics is matched with the same $\beta^{*}$ in both planes. Under these conditions, the solutions (15) and (16) take the following simple form:

$$
\begin{aligned}
& \Delta K_{2_{F}}^{L, R}=\frac{1}{N_{F}^{L, R} \beta_{F} D_{x_{F}}} \times \frac{I_{0}}{\sqrt{1+r_{F D}^{2}}}, \\
& \Delta K_{2_{D}}^{L, R}=-\frac{1}{N_{D}^{L, R} \beta_{F} D_{x_{D}}} \times \frac{I_{0}}{\sqrt{1+r_{F D}^{2}}},
\end{aligned}
$$

and

$$
\begin{aligned}
& \exp \left[2 j \Delta \mu_{x}^{L, R}\right]=-\frac{1}{\sqrt{1+r_{F D}^{2}}}-j \frac{\xi^{L, R} r_{F D}}{\sqrt{1+r_{F D}^{2}}}, \\
& \exp \left[2 j \Delta \mu_{y}^{L, R}\right]=-\frac{1}{\sqrt{1+r_{F D}^{2}}}+j \frac{\xi^{L, R} r_{F D}}{\sqrt{1+r_{F D}^{2}}},
\end{aligned}
$$

with

$$
I_{x}=I_{y} \stackrel{\text { def }}{=} I_{0} \quad \text { when } \quad \beta_{x}^{*} \equiv \beta_{y}^{*} \stackrel{\text { def }}{=} \beta_{0}^{*} .
$$

Note that Eq. (20) is also equivalent to the following relation:

$$
\begin{aligned}
\cot \left[\Delta \mu_{x, y}^{L, R}\right] & \equiv \mp \frac{\xi^{L, R} r_{F D}}{\sqrt{1+r_{F D}^{2}}+1} \\
& \text { or } \Delta \mu_{x, y}^{L, R} \equiv \frac{\pi}{2} \bmod [\pi] \pm \tan ^{-1}\left[\frac{\xi^{L, R} r_{F D}}{\sqrt{1+r_{F D}^{2}}+1}\right],
\end{aligned}
$$

where the \pm sign in the second relation stands for the horizontal and vertical plane, respectively.

The optimal left and right IR phases are then relatively close, but not exactly equal to $\pi / 2$, with a deviation amounting to about $\pm r_{F D} / 2= \pm 1.6 \times 10^{-2} \times 2 \pi \approx \pm 6^{\circ}$. This phase shift may look small, but has been found fundamental in order to preserve the quality of the chromatic correction during the telescopic part of the squeeze towards very low $\beta^{*}$.

Finally, the sextupole settings obtained in Eq. (19) can obviously not warrant at the same time an exact compensation of the linear chromaticity. Nevertheless, as can be seen by inserting (19) into (11), the contribution of the inner triplets to the linear chromaticity is then strongly reduced, by a factor close to $6\left(\sim 1 / r_{F D}\right)$ after the offmomentum $\beta$-beating correction. The strategy is then to apply a global chromaticity correction, keeping constant the sextupole settings in the arcs which are directly adjacent to the low- $\beta$ insertions (namely the arcs $45,56,81$, and 12), and using all the focusing and defocusing sextupoles located in the other arcs (namely the arcs 23, 34, 67, and 78), with the families (a) and (b) powered in series in this case. As a result, if the betatron phase advances per cell are also relatively close to $\pi / 2$ in these arcs, the correction of the off-momentum $\beta$ beating formerly established will be rather insensitive to the final adjustments of chromaticity. This "impurity" of the presqueezed optics will however rapidly disappear during the telescopic part of the squeeze (see Appendix A).

\section{The construction of the telescopic optics and its chromatic correction}

One of the most remarkable features of the ATS scheme is that the second (telescopic) part of the squeeze can be performed at quasiconstant settings in all the sextupoles of the lattice. In order to understand this property, the machinery deployed during this process shall first be presented in detail. Starting from a round presqueezed optics $\left(\beta_{x}^{*} \equiv\right.$ $\beta_{y}^{*}=\beta_{0}^{*}$ ) fulfilling the phasing conditions given in Eqs. (10) and (22), and targeting a smaller $\beta^{*}$ for the collision optics, round or flat, and defined with a new set of IP parameters $\left(\tilde{\beta}_{x, y}^{*}, \alpha_{x, y}^{*} \equiv 0, D_{x}=D_{x}^{\prime} \equiv 0\right)$, the telescopic squeeze consists in the two following operations. First, the new IP parameters are backtracked through the arc on the left side of the IP (e.g. from IP5 through arc 45), until reaching the first insertion upstream of the IP (e.g. IR4 for squeezing IR5). This "left supporting IR" is then rematched to the new boundary conditions on its right, preserving in addition the overall betatron phase advances from its entry to the IP (e.g. from Q13.L4 to IP5 in Fig. 4). This technique is repeated on the right side, tracking the new IP parameters until reaching the next insertion downstream of the IP (e.g. IR6 for squeezing IR5), and rematching the "right supporting" insertion to the new boundary conditions on its left, still keeping constant the betatron phase advances over the full section (e.g. from IP5 to Q13.R6). In this process the settings of the matching quadrupoles in the high luminosity insertions proper are kept strictly constant, together with the arc quadrupoles (as for any standard squeeze). While the horizontal dispersion function remains clearly periodic in the arcs during the telescopic squeeze, the $\beta$ functions are mismatched in the 
two arcs on either side of the IP, in fact increasing roughly as $1 / \tilde{\beta}^{*}$ at the location of the strong sextupoles (see Appendix A):

$$
\frac{\tilde{\beta}_{F_{x}}}{\beta_{F_{x}}} \approx \frac{\beta_{0}^{*}}{\tilde{\beta}_{x}^{*}} \quad \text { and } \quad \frac{\tilde{\beta}_{D_{y}}}{\beta_{D_{y}}} \approx \frac{\beta_{0}^{*}}{\tilde{\beta}_{y}^{*}},
$$

where, from now on, any quantity marked with a tilde keeps the same meaning as before, but will refer to the perturbed (telescopic) optics. Therefore, the efficiency of the strong sextupoles increases at constant strength during the telescopic squeeze, and exactly in proportion with the increase of the chromatic aberrations induced by the final focus quadrupoles [see Eq. (4)]. As a result, for any collision $\tilde{\beta}^{*}$ which of course can be matched by the left and right supporting IRs, the chromatic correction of the inner triplet only requires a small readjustment of the strong sextupole families with respect to the settings established previously in Eq. (19) for the presqueezed optics (see Appendix A for more details):

$$
\begin{aligned}
& \Delta \tilde{K}_{2_{F}}^{L, R}=\lambda \times \Delta K_{2_{F}}^{L, R}=\frac{\lambda}{N_{F}^{L, R} \beta_{F} D_{x_{F}}} \times \frac{I_{0}}{\sqrt{1+r_{F D}^{2}}}, \\
& \Delta \tilde{K}_{2_{D}}^{L, R}=\lambda \times \Delta K_{2_{D}}^{L, R}=-\frac{\lambda}{N_{D}^{L, R} \beta_{F} D_{x_{D}}} \times \frac{I_{0}}{\sqrt{1+r_{F D}^{2}}},
\end{aligned}
$$

where all the quantities in the right-hand side (rhs) refer to the unperturbed (presqueezed) optics, and the scaling factor $\lambda$ is given by

$$
\lambda \sim \frac{1}{1-\kappa\left[1-\left(\frac{\tilde{\beta}^{*}}{\beta_{0}^{*}}\right)^{2}\right]} \sim 1,
$$

with

$$
\kappa \stackrel{\text { def }}{=} \frac{1}{2}\left[1-\frac{1-r_{F D}}{\sqrt{1+r_{F D}^{2}}}\right] \sim \frac{r_{F D}}{2} .
$$

\section{Nonlinear chromaticity}

One important remaining item concerns the nonlinear chromaticity, and in particular the second order chromaticity $Q^{\prime \prime}$. The latter may not be automatically compensated by the chromatic correction strategy discussed in the previous sections. Neglecting the contribution of the second order dispersion induced by the strong sextupoles in the arcs, which is a valid approximation for $\pi / 2$ lattices, the second order chromaticity can be written as follows (see for instance [3]):

$Q_{x, y}^{\prime \prime} \approx \mp \frac{1}{4 \pi} \int_{\text {Ring }} d s\left[K_{1}(s)-K_{2}(s) D_{x}(s)\right]\left(\partial_{\delta} \beta_{x, y}\right)_{(\delta=0 ; s)}$,

where the first order chromatic derivative of the $\beta$ functions is given by

$$
\begin{aligned}
& \left(\partial_{\delta} \beta_{x, y}\right)_{(\delta=0 ; s)} \\
& = \pm \frac{\beta_{x, y}(s)}{2 \sin \left(2 \pi Q_{x, y}\right)} \int_{0}^{C} d s_{1}\left[K_{1}\left(s_{1}\right)-K_{2}\left(s_{1}\right) D_{x}\left(s_{1}\right)\right] \\
& \quad \times \beta_{x, y}\left(s_{1}\right) \cos \left[2\left|\mu_{x, y}\left(s_{1}\right)-\mu_{x, y}(s)\right|-2 \pi Q_{x, y}\right] .
\end{aligned}
$$

Since the off-momentum $\beta$ beating, $\partial_{\delta} \beta_{x, y}$, induced by the strong sextupoles arrives out of phase by $\pi / 2$ at the location of the final focus quadrupoles $\left(K_{1} \partial_{\delta} \beta \sim 0\right)$, the contribution of the low- $\beta$ insertions to $Q^{\prime \prime}$ is expected to be rather small. Therefore, neglecting the contribution of the other quadrupoles and of the weak sextupole families of the ring, the integral defining $Q^{\prime \prime}$ can be seen as a double sum over the strong sextupoles only, involving cross talks between the off-momentum $\beta$ beating induced by one strong SF or SD of the lattice and interacting with the chromatic quadrupole error $\Delta K_{2} D_{x}$ induced by another strong sextupole. These cross talks only concern the strong sextupoles installed in the same arc since, by construction of ATS optics, the offmomentum $\beta$-beating wave induced by one full arc of sextupoles is vanishing after its passage in the final focus quadrupoles. In addition, there are no cross talks between two strong sextupoles of the same family (SF or SD), since the latter are spaced by $0 \bmod [\pi]$ in betatron phase (both for the presqueezed and the telescopic optics). Indeed, the offmomentum $\beta$ beating induced by a given strong sextupole arrives always out of phase by $\pi / 2$ in all the strong sextupoles of its own family, and therefore cannot contribute to $Q^{\prime \prime}$. The only potential source of $Q^{\prime \prime}$ is therefore expected from the cross talks between the strong SFs and strong SDs. On the other hand, most of these cross talks are in fact selfcompensated two by two, both for the presqueezed and the telescopic optics. Indeed, let us consider first the presqueezed optics, and assume for example that the left arc starts with a strong SF, with $\Delta \mu_{F D}=\pi / 4$ for the betatron phase advance until the first SD. The first SF will generate a horizontal off-momentum $\beta$ beating which will reach its maximum at $\pi / 4$ downstream in phase, which is at the location of the first strong SD. This cross talk will contribute to $Q_{x}^{\prime \prime}$ as follows:

$$
\Delta Q_{x}^{\prime \prime}=-\xi \frac{1}{4 \pi} \Delta K_{2_{F}}^{L} \Delta K_{2_{D}}^{L} \beta_{F} \beta_{D} D_{x_{F}} D_{x_{D}},
$$

with $\xi=\sin \left(2 \Delta \mu_{F D}\right)=1$ in this example [see also Eq. (14) for the general case]. The first strong SD will in turn generate a horizontal off-momentum $\beta$ beating which, contrary to the SF of above, will reach its minimum at the location of the second SF, at $3 \pi / 4$ downstream in betatron phase. Its contribution in terms of $Q^{\prime \prime}$ will therefore exactly compensate the first one, and so on and so forth. The situation can then be analyzed in a similar way, and the same conclusions can be drawn for the telescopic optics, although the $\pi / 4$ (or $3 \pi / 4$ ) phase advance between two consecutive SF and SD is broken in this case, but not the phase advance of $\pi$ between two strong sextupoles belonging to the same family. As a result, only end effects might give a systematic contribution 
to $Q^{\prime \prime}$, due to the finite number of strong SFs and SDs in each arc, even if their number is the same in a given arc $\left(N_{F} \equiv N_{D}=N\right)$. Indeed coming back to the above example of an arc starting with a strong focusing sextupole, the latter will contribute $N$ times to $Q^{\prime \prime}$ via its consecutive cross talks with the $N$ defocusing sextupoles located downstream. On the other hand, the first strong SD of this arc will only interact $N-1$ times with the strong SF family, preventing consequently an exact compensation of the first contribution. Although marginal for the presqueezed optics, this contribution to $Q^{\prime \prime}$ nevertheless grows quadratically with $1 / \beta^{*}$ during the telescopic squeeze. An elegant solution consists in a very fine-tuning of the left and right phase advances of the low- $\beta$ insertions for a global correction of $Q^{\prime \prime}$, at a cost of regenerating a small off-momentum $\beta$ beating in the final focus quadrupole and at the IP, but not exceeding the percent level as we will see in the next section.

\section{Final remarks}

The situation might be slightly more complicated in a real machine, for various reasons, and in particular for the LHC, with some of the strong sextupoles installed in the dispersion suppressors where the $\beta$ functions and the betatron phase advances do not coincide exactly with those of the regular arc. Moreover, some contributions to the chromaticity and off-momentum $\beta$ beating are expected from the matching quadrupoles equipping the low- $\beta$ insertions (not only the inner triplet), and also from those of the other IRs which are involved in the telescopic squeeze. However, as it will be illustrated in the next section, all these contributions have been found marginal for the LHC, even pushing $\beta^{*}$ down to extreme values with the ATS, such as $\beta_{x, y}^{*}=10 \mathrm{~cm}$ for round telescopic optics, or even $\beta_{x, y}^{*}=5 / 20 \mathrm{~cm}$ for flat optics, which is a factor of 5 to 10 below the $55 \mathrm{~cm}$ nominal $\beta^{*}$ of the LHC [1]. More precisely, as soon as proper MADX [11] tools were built to properly implement the ATS principles, and finally finetune the chromatic properties of the telescopic optics in terms of chromaticity $Q_{x, y}^{\prime}$ or Montague functions $W_{x, y}$, the numerical results obtained have always perfectly met the expectations derived analytically in the previous sections.

Finally, mismatching the $\beta$ functions in the arcs is of course not completely without any risk, not so much in terms of mechanical acceptance at flattop energy as already mentioned, but possibly in terms of dynamic aperture. This point will be briefly discussed in the next section and relevant references provided accordingly.

\section{ATS OPTICS IN PRACTICE ILLUSTRATED WITH THE (HL)-LHC}

\section{A. The development of the ATS scheme over the past few years}

The basic principles of the achromatic telescopic squeezing scheme were first established in 2010, together with a series of new MADX tools in order to build accordingly a full set of presqueezed and telescopic collision optics for the LHC ring [8]. The first ATS optics were constructed assuming the new $120 \mathrm{~mm}$ aperture $\mathrm{Nb}-\mathrm{Ti}$ triplet $(120 \mathrm{~T} / \mathrm{m})$ which was proposed for the former upgrade project of the LHC, the so-called LHC upgrade project phase I [12]. The phase I project was then replaced later by the wider scope HL-LHC project [2]. During this transition period, the ATS principles were demonstrated with the effective construction and chromatic correction of flat telescopic optics, where $\beta^{*}$ was squeezed down to $30 \mathrm{~cm}$ in the crossing plane and pushed to $7.5 \mathrm{~cm}$ in the order plane [8]. The well-known strategy behind flat optics was to mitigate the loss of luminosity in the presence of a nonzero crossing angle. The feasibility of such low- $\beta^{*}$ optics was however not at all expected at that time for the LHC. In parallel a full set of specifications was also established for the list and the mechanical aperture of the magnets to be replaced in order to fully exploit the new possibilities offered by ATS optics: not only the inner triplet, where the new target aperture of $150 \mathrm{~mm}$ was derived [8] (and recently adopted in the baseline of the HL-LHC Project), but also the separation-recombination dipoles D1 and D2, and the first two quadrupoles of the matching section Q4 and Q5 (see Fig. 2). Then, round telescopic optics with a $\beta^{*}$ squeezed down to $15 \mathrm{~cm}$ in both planes [13] were also made available, still based on the phase I triplet layout, but in an upgrade scenario relying on the presence of crab cavities [14] which became as well a key ingredient of the HL-LHC project.

\section{ATS and dynamic aperture}

A potential severe limitation to the ATS scheme was however its negative impact onto the dynamic aperture, driven by the increase of the peak $\beta$ functions in the arcs during the telescopic squeeze, the nonlinear field imperfections of the arc magnets, and the third order resonance driving terms excited by the lattice sextupoles involved in the chromatic correction. On the other hand, thanks to the tremendous efforts which were deployed for the LHC main magnets during the construction and installation phases of the machine, both in terms of field quality specifications [15] and monitoring [16,17], and in terms of sorting strategy (see e.g. [18] and reference therein), the existing machine was found compatible with the ATS scheme for this aspect, although not designed for it. Indeed, (i) within the limits reported in Figs. 4(b) and 4(c) for the increase of the peak $\beta$ functions in the arcs (i.e. a factor of 4 in both planes for round telescopic optics, and $2 / 8$ in the $\mathrm{H} / \mathrm{V}$ or $\mathrm{V} / \mathrm{H}$ planes for flat optics), (ii) considering only in a first step the field imperfections of the arc magnets as measured and installed in the existing LHC ring, (iii) and ensuring that the strong sextupole families do contain an even number of magnets (for a two-by-two compensation at $\pi$ of their contribution to the third order resonance driving 
terms), the (HL)-LHC dynamic aperture was found to be about 40 beam $\sigma$ for the presqueezed optics, then dropping to $15 \sigma$ and $11 \sigma$ for the telescopic round and flat optics corresponding to Figs. 4(b) and 4(c), respectively [13,19]. On the other hand, assigning realistic field imperfections to the new HL-LHC magnets, the HL-LHC dynamic aperture was found to be driven anyway by the field quality of the new triplets $[20,21]$ (reaching about $10-11 \sigma$ and $6-7 \sigma$ for typical round and flat ATS telescopic optics, with $\beta_{x, y}^{*}=$ $15 \mathrm{~cm}$ and $\beta_{x, y}^{*}=30 / 7.5 \mathrm{~cm}$, respectively). This aspect will therefore no longer be addressed in all the rest of the paper, but keeping in mind that item (iii) above implies the installation of an additional sextupole at Q10 in the IR1 and IR5 insertions of the LHC.

\section{Modularity of the ATS versus triplet layout}

With the exception of the Q5 matching quadrupoles of IR6 which would need to be made $20 \%$ stronger or longer in order to achieve the telescopic squeeze of IR5 at $7 \mathrm{TeV}$, and provided heavier interventions in IR1 and IR5 to maximize the mechanical acceptance of the new high luminosity insertions, the ATS scheme was then found to be fully compatible with the existing hardware and layout of the LHC [8]. Step by step, this scheme was pushed in order to produce and ensure the chromatic correction of collision optics with extremely small $\beta^{*}$, round or flat, down to $\beta_{x, y}^{*}=10 \mathrm{~cm}$ or $\beta_{x, y}^{*}=5 / 20 \mathrm{~cm}$, respectively.

ATS optics were built in practice assuming several possible triplet layouts for the new high luminosity insertions, with an operating gradient $G$ ranging from 100 to $200 \mathrm{~T} / \mathrm{m}$ [8,22-24]. Amongst these various cases, a completely new version of the LHC optics was also developed based on the ATS scheme, and compatible with the existing layout of the machine, until an energy of about $6 \mathrm{TeV}$ per beam (limited by the strength of the Q5 quadrupoles in IR6). The aim was to prepare dedicated machine experiments and achieve a series of validation tests with beam of the ATS principles, until successively demonstrating the feasibility of a $10 \mathrm{~cm}$ $\beta^{*}$ collision optics for the HL-LHC (in very specific configurations, of course not suitable for nominal operation). The results of these beam experiments have been documented in a series of CERN technical notes [25-28] and will form the content of a separated publication.

As expected, the difference between all these cases was found really minor in terms of optics, but of course not in terms of layout. It concerns essentially the minimum possible presqueezed $\beta^{*}$, which was found limited by the maximum strength available in the arc sextupoles, ranging in between 36 and $50 \mathrm{~cm}$ with again a rough scaling like $\beta_{\text {presqueeze }}^{*} \propto 1 / \sqrt{G}$ [see also Eq. (1) in another context].

In order to reach smaller $\beta^{*}$, the telescopic techniques of the ATS were then deployed, with some modularity properties which are important to emphasize. Indeed, for two different possible operating gradients $G_{1,2}$ (and therefore layout and aperture) for the new triplets in IR1 and IR5, and two corresponding presqueezed optics matched to $\beta_{\text {presqueeze }_{1,2}}^{*}$ at IP1 and IP5, any settings assigned to the matching quadrupoles of IR2, IR8, IR4, and IR6 in order to produce a telescopic optics with $\beta^{*}=\beta_{\mathrm{col}_{1}}$ for the first layout could then be directly reused in order to generate a perfectly matched telescopic optics for the second layout also, with

$$
\frac{\beta_{\mathrm{col}_{1}}^{*}}{\beta_{\mathrm{col}_{2}}^{*}} \equiv \frac{\beta_{\text {presqueeze }_{1}}^{*}}{\beta_{\text {presqueeze }_{2}}^{*}} \propto \sqrt{G_{2} / G_{1}} .
$$

The only condition was to match these various presqueezed optics with always the same left and right phase advances in IR1 and IR5, as prescribed in Eq. (22). This modularity facilitated substantially the study and the design of the HL-LHC optics and layout.

Consequently, and unless specified differently in the following, the existing LHC layout, and in particular the existing inner triplet with a nominal gradient of $205 \mathrm{~T} / \mathrm{m}$, will be used as reference in order to illustrate the ATS presqueezed and telescopic optics, together with their chromatic properties. The situation would indeed not change qualitatively for the HL-LHC or any other triplet layout operating at lower gradient $G$ (compatible with larger aperture), only quantitatively in terms of minimum possible presqueezed $\beta^{*}$, or relative increase of the peak $\beta$ functions in the arcs for a given $\beta^{*}$ in collision, which would slightly increase with $1 / \sqrt{G}$.

\section{B. ATS optics for the (HL-)LHC \\ 1. The injection optics}

Generally speaking, an injection optics is an optics which provides a certain number of functionalities in the various insertions of the ring (e.g. the injection of Beam1 and Beam2 through IR2 and IR8 as shown in Fig. 1), while being compatible with the physical emittances of the injected beams in terms of mechanical acceptance. Concerning the experimental insertions in particular, the injection $\beta^{*}$ shall therefore be relaxed with respect to its value at collision, typically by a factor corresponding to the dynamic energy range of the machine. On the other hand, keeping in mind the ATS scheme which will be deployed at flattop energy, some specific additional constraints shall already be taken into account when preparing the injection optics.

(a) Arc optics.-First of all, as explained in Sec. II B 1, the phase advances per cell need to be matched to $\pi / 2$ in the arcs on either side of the high luminosity insertions:

$$
\Delta \mu_{x, y}^{\mathrm{cell}} \equiv \frac{\pi}{2} \text { in the } \operatorname{arcs} 81,12,45, \text { and } 56 .
$$

(b) High luminosity insertions.-The phase advances over the low- $\beta$ insertions proper are kept constant during the presqueeze (as for any standard squeeze) in order to keep the overall tune unchanged during this process (see Sec. IB 2). Combining this request with the phasing 
properties of the presqueezed optics [see Eq. (22)], it is clear that the low- $\beta$ insertions shall be matched with an overall phase advance of about $\pi \bmod [\pi]$ in both planes at injection, more precisely in between the last strong SF (respectively SD) sextupole on the left side of the IP, and the first one on the right side. For the LHC conventions, this requirement corresponds to

$$
\Delta \mu_{x, y}^{\mathrm{IR} 1} \equiv \Delta \mu_{x, y}^{\mathrm{IR} 5} \approx \frac{\pi}{4}\left[\frac{\pi}{2}\right],
$$

since the horizontal and vertical phase advances over the insertions are defined from Q13.L to Q13.R (see Sec. I B 2), with Q13.L/R being of opposite polarity (antisymmetric optics). The above conditions are actually defined within a multiple integer of $\pi / 2$, which just illustrates the fact that the strong sextupole families can a priori be arbitrarily selected amongst the available families "a" and "b" (see Fig. 3). In practice, this choice might however be strongly limited by the tunability in phase of the low- $\beta$ insertions at small $\beta^{*}$. As a result, all the ATS optics developed so far for the LHC were matched with an overall phase advance of about $\Delta \mu_{x, y}^{\mathrm{IR} 1, \mathrm{IR} 5} \approx 2.625 \times 2 \pi$, corresponding to the following left and right phase advances for the presqueezed optics:

$$
\begin{aligned}
& \Delta \mu_{x_{\text {Beaml }}, y_{\text {Beam } 2}}^{\mathrm{Q} 13 \mathrm{IP}} \approx 1.125 \times 2 \pi, \quad \Delta \mu_{x_{\text {Beaml }}, y_{\text {Beam } 2}}^{\mathrm{IP} \rightarrow \mathrm{R} 13 . \mathrm{R}} \approx 1.5 \times 2 \pi, \\
& \Delta \mu_{y_{\text {Beaml }}, x_{\text {Beam } 2}}^{\mathrm{Q} 13, \mathrm{IP}} \approx 1.5 \times 2 \pi, \quad \Delta \mu_{y_{\text {Beal } 1}, x_{\text {Beam } 2}}^{\mathrm{IP} \rightarrow \mathrm{Q} 13 . \mathrm{R}} \approx 1.125 \times 2 \pi,
\end{aligned}
$$

which are compatible with the condition (22) provided that the first strong SFs (respectively SDs) for Beam1 on either side of the IP are selected at Q10.L/Q11.R, then Q14.L/ Q15.R, ... (respectively Q11.L/Q10.R, then Q15.L/Q14.R, ...), and conversely for Beam2 (see Fig. 4 illustrating the case of Beam1 on the left side of IP5).

(c) Other insertions. - In view of the telescopic part of the squeeze, the injection optics shall also reserve enough provision for the normalized strength of the matching quadrupoles equipping the left and right supporting insertions (IR8, IR2, IR4, and IR6), but without compromising their other (non-ATS) functionalities, and of course preserving their mechanical acceptance at injection. Within the exception of the Q5 quadrupoles of IR6 (see Sec. III A 2), this request does not require any additional hardware modifications in the LHC ring. It was also not found to be a serious constraint for redefining the injection optics, thanks to the rather generous number of individually powered quadrupoles which equip the eight insertions of the ring.

(d) Betatron tunes.-Finally, the fractional part of the betatron tunes can be easily recovered by retuning accordingly the settings of the main quadrupoles located in the arcs which do not participate to the telescopic squeeze (namely the arcs 23, 34, 67, and 78). In the end of this process, the integer tunes are not necessarily preserved (e.g. $Q_{x, y}=64.28 / 59.31$ for the nominal injection optics of the LHC, compared to 62.28/60.31 for the ATS injection optics developed so far).

\section{The presqueezed optics}

One of the keystones of the ATS scheme is the presqueezed optics, with specific matching conditions given for the left and right phase advances of the high luminosity insertions [see the relations (33)]. The LHC low- $\beta$ insertions were however not designed for these two additional matching constraints. As a result the presqueezed $\beta^{*}$ can only be chosen within a relatively tight interval. This interval depends on the detailed layout and gradient of the final focus quadrupoles, on the maximum operating current of the lattice sextupoles and on the beam energy. At nominal energy (7 TeV/beam) and for the existing LHC triplet $(205 \mathrm{~T} / \mathrm{m})$, the presqueezed $\beta^{*}$ shall satisfy the following conditions:

$$
36-40 \mathrm{~cm} \lesssim \beta_{\text {presqueezed }}^{*} \leq 2-3 \mathrm{~m} .
$$

Indeed, the IR phasing conditions mentioned above cannot be sustained for a presqueezed $\beta^{*}$ larger than 2-3 m, because of strength limitations which are observed in some quadrupoles of the matching section, in particular Q6 approaching its nominal current. Moreover, a $\beta^{*}$ of only $2 \mathrm{~m}$ would already be too small for the injection optics, because it would be incompatible with the aperture requirements at injection energy, both for the existing LHC triplets and the new triplets of larger aperture foreseen for the HL-LHC. This limitation is however a nonissue since the chromatic aberrations are completely marginal above a $\beta^{*}$ of a few meters in the LHC. On the other hand, this somehow complicated the optimization process of ATS optics, imposing a kind of iterative process between the injection and the presqueezed optics.

On the opposite side, below a presqueezed $\beta^{*}$ of about $40 \mathrm{~cm}$, some matching quadrupoles of IR 1 and IR5 start to be pushed to rather low gradients (in particular Q6) while others, like Q7 in the dispersion suppressor, are approaching their nominal field. Moreover, for a too low presqueezed $\beta^{*}$, some strong defocusing sextupole families would need to be powered beyond their maximum current of $600 \mathrm{~A}$. With the existing sextupole layout of the LHC, the minimum possible presqueezed $\beta^{*}$ has then been found to be $40 \mathrm{~cm}$ for the existing inner triplet. Assuming the installation of an additional arc sextupole at Q10 in IR1 and IR5, as requested for the HL-LHC for preserving the dynamic aperture of the telescopic optics (see Sec. III A 1), the minimum possible presqueezed $\beta^{*}$ can be pushed down to $36 \mathrm{~cm}$. This value can be directly compared with a minimum possible presqueezed $\beta^{*}$ of $44 \mathrm{~cm}$ which is obtained for the baseline $(140 \mathrm{~T} / \mathrm{m}-150 \mathrm{~mm})$ inner triplet of the HL-LHC [24], which exactly follows the scaling law formulated in Eq. (30). 
TABLE I. Minimum possible presqueezed $\beta^{*}$ in various cases for the LHC and HL-LHC at an energy 7 TeV/beam (aperture requirements not included in the case of the existing LHC triplets).

\begin{tabular}{lccc}
\hline \hline Case & 1 & 2 & 3 \\
\hline Triplet & LHC (205 T/m) & LHC (205 T/m) & HL-LHC (140 T/m) \\
Sextupole layout & Nominal (11 strong SDs/arc) & New (12 strong SDs/arc) & New (12 strong SDs/arc) \\
Sextupole current [A] & 600 & 600 & 600 \\
Minimum presqueezed $\beta^{*}[\mathrm{~cm}]$ & 40 & 36 & 44 \\
Peak $\beta$ in triplet $[\mathrm{km}]$ & 6.0 & 6.6 & 7.0 \\
\hline \hline
\end{tabular}

These various cases are summarized in Table I, and the corresponding presqueezed optics illustrated in Figs. 5(a)-5(c). These optics can then be compared to an extreme case where the nominal squeeze sequence of the LHC has been pushed down to $\beta^{*}=35 \mathrm{~cm}$ at IP1 and IP5 [29] [see Fig. 5(d)], with some matching quadrupoles already slightly beyond the limits, and a "minimal" chromatic correction limited to a global correction of the linear chromaticity. In all cases, the peak $\beta$ functions $\beta_{\max }$ reached in the triplets follow rather precisely the scaling law given in Eq. (1). In the case of the ATS presqueezed optics [Figs. 5(a)-5(c)], the slope of the $\beta$ functions at the triplet exit is however more pronounced in the defocusing plane of Q3, leading in particular to substantially smaller $\beta$

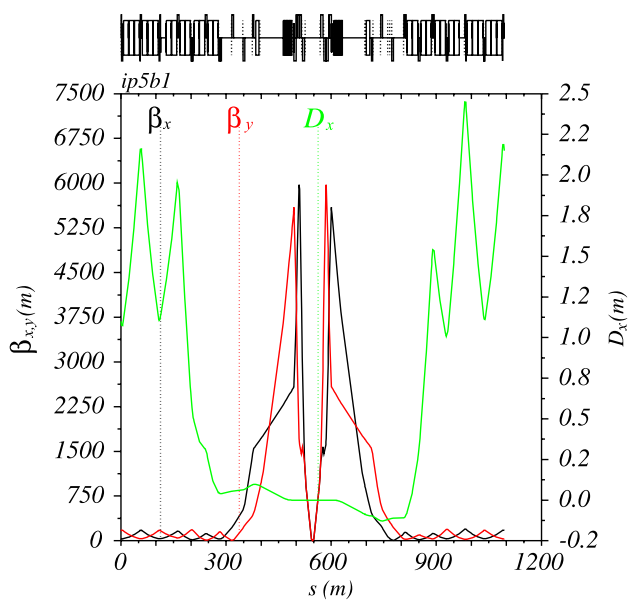

(a): $\beta^{*}=40 \mathrm{~cm}($ Case 1 of Tab. I)

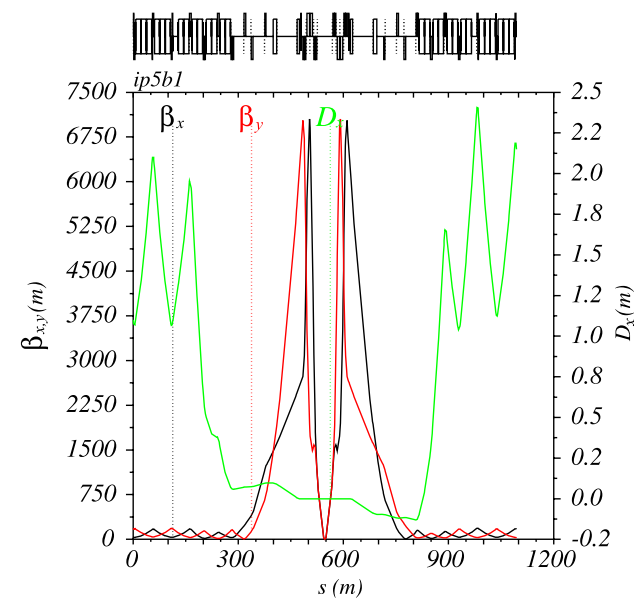

(c): $\beta^{*}=44 \mathrm{~cm}($ Case 3 of Tab. I)

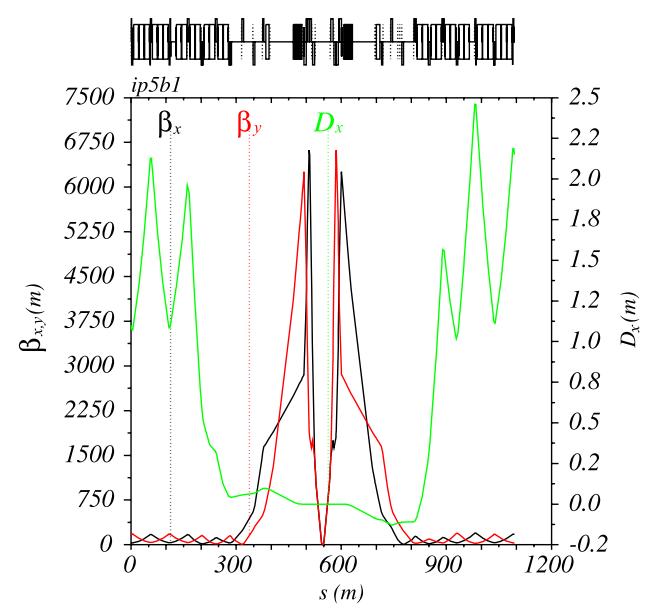

(b): $\beta^{*}=36 \mathrm{~cm}($ Case 2 of Tab. I)

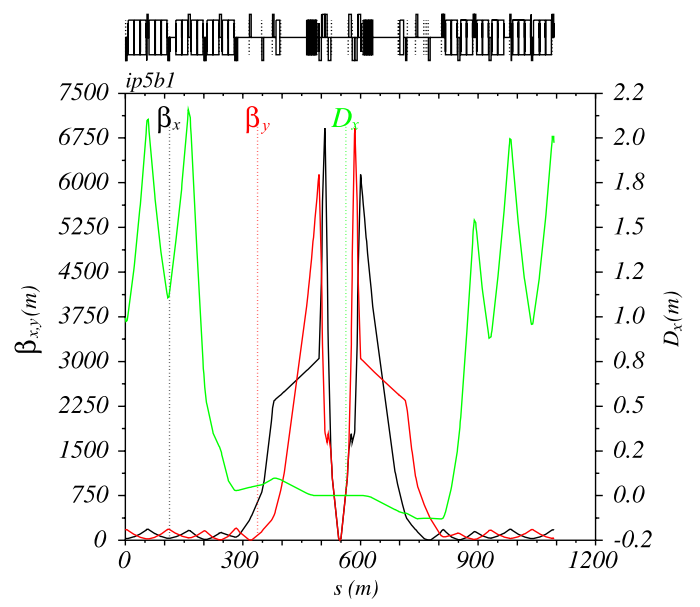

(d): $\beta^{*}=35 \mathrm{~cm}$ ("nominal-like LHC optics")

FIG. 5. Presqueezed ATS optics for Beam1 zoomed in the CMS experimental insertion for the three cases summarized in Table I [(a)-(c)], and compared with a nominal-like LHC collision optics (d), which has been already pushed beyond the gradient limits of some IR quadrupoles in order to reach $\beta^{*}=35 \mathrm{~cm}$. The crossing scheme is assumed to be switched off in order to preserve the dispersion matching (see also Sec. III C). 
functions at $\mathrm{Q} 4$ for the same $\beta_{\max }$ reached in the inner triplet. This feature is the signature of the additional matching constraints which are imposed on the left and right phase advances of the low- $\beta$ insertions in the ATS case (and are achieved in practice by acting on a small trim power supply feeding Q1 in the LHC).

The difference between ATS presqueezed and nominallike collision optics is even more striking when comparing their chromatic properties at very similar $\beta^{*}\left(\beta^{*}=36 \mathrm{~cm}\right.$ and $\beta^{*}=35 \mathrm{~cm}$ in the first and second case, respectively). As illustrated in Fig. 6(a), the chromatic variations of the betatron tunes are very linear for ATS optics, following the slope of the linear chromaticity $Q^{\prime}$ which has been matched to two units in this case. Under the same conditions, a strong nonlinear chromaticity shows up in the second case [see Fig. 6(b)]. Then, in the first case, the chromatic

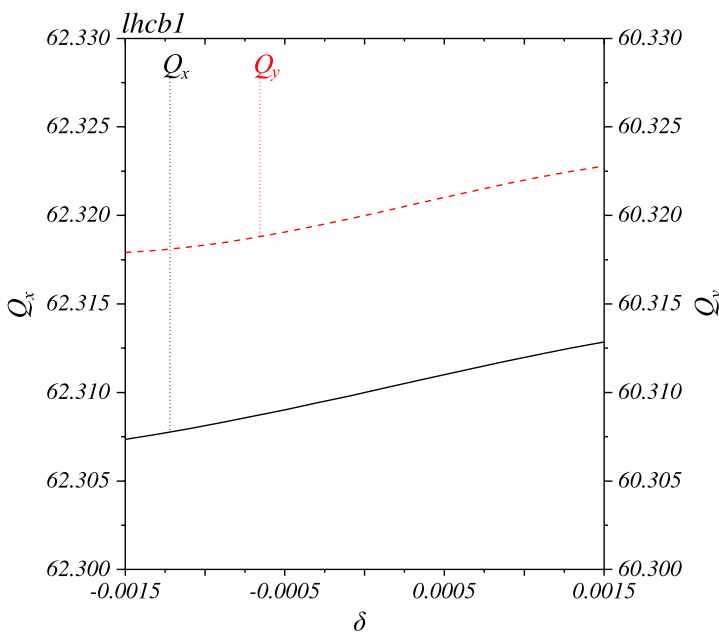

(a): $Q_{x, y}(\delta)$ (pre-squeezed ATS optics)

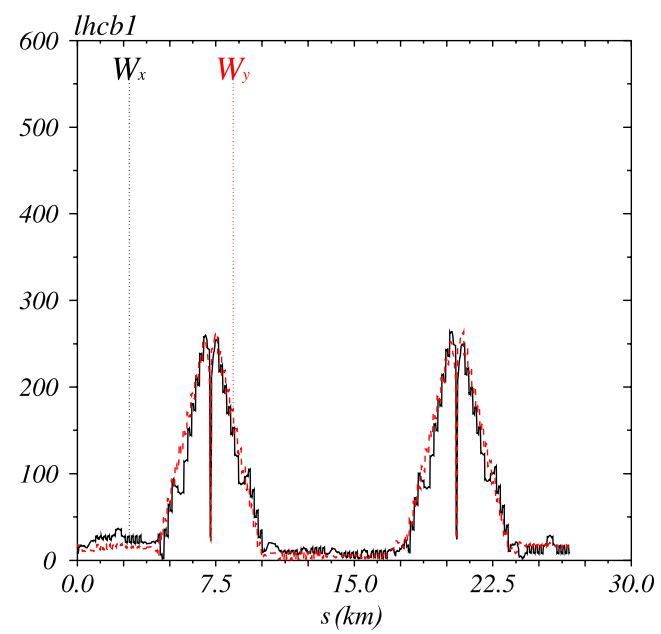

(c): $W_{x, y}(s)$ (pre-squeezed ATS optics)
Montague functions $W_{x, y}$ are nicely vanishing beyond the two arcs on either side of the low- $\beta$ insertions, in particular in the collimation insertions IR3 and IR7 [see Fig. 6(c)]. Still for the ATS case, the $W$ functions reach their maximum in the triplet, while they are minimal at the IPs of the two high luminosity experiments, corresponding to the quantities $I_{x, y}$ and $\Delta I_{x, y}$, respectively, defined in Eq. (6). Indeed, by construction of the presqueezed optics, a wave of off-momentum $\beta$ beating is coherently excited by the strong sextupoles of the arcs on either side of IP1 and IP5. This wave arrives out of phase by $\pi / 2$ at the location of the inner triplets for an in-phase compensation of the chromatic kick induced by the low- $\beta$ quadrupoles. After its passage in the triplet, this wave is then strictly vanishing at the IP in terms of (first order) off-momentum $\beta$ beating, but not completely in terms of off-momentum

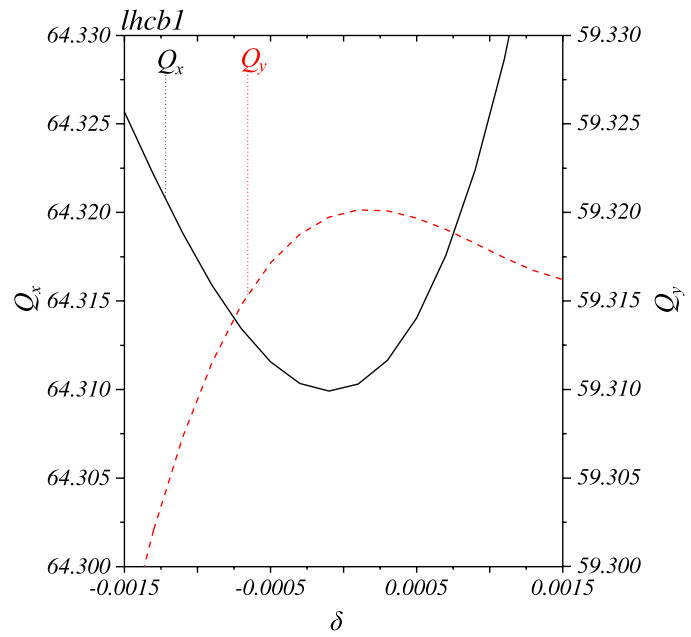

(b): $Q_{x, y}(\delta)$ (nominal-like LHC optics)

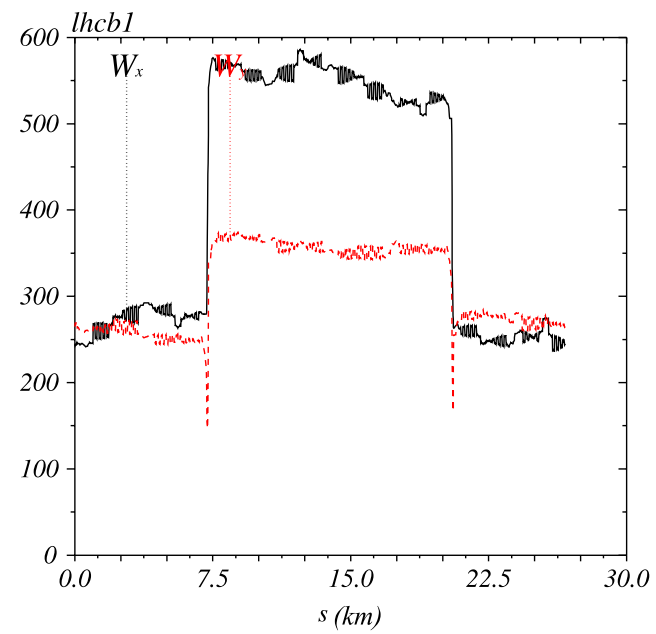

(d): $W_{x, y}(s)$ (nominal-like LHC optics)

FIG. 6. Chromatic variations of the betatron tunes (top) and Montague functions (bottom) along the LHC ring (cycled from IP3). The left pictures stand for an ATS optics presqueezed down to $\beta^{*}=36 \mathrm{~cm}$ at IP1 and IP5 (see case 2 of Table I), which is compared to a nominal-like LHC collision optics with $\beta^{*}=35 \mathrm{~cm}$ (right pictures). 
$\alpha$ beating $\left(\Delta I_{x, y} \neq 0\right.$ for the antisymmetric LHC optics). The situation is qualitatively and quantitatively very different for the nominal-like collision optics, as shown in Fig. 6(d).

Looking more into the details of the chromatic behavior of the Twiss parameters at specific locations in the LHC ring, in particular at the interaction point of the CMS experiment (IP5) or in the betatron collimation insertion (IR7), variations of the $\beta$ functions by up to $100 \%$ can be observed over a momentum window of $\pm 1.510^{-3}$ for the nominal-like collision optics with $\beta^{*}=35 \mathrm{~cm}$. This can be directly compared to the few percent variations obtained for the ATS optics presqueezed down to $\beta^{*}=36 \mathrm{~cm}$ (see Fig. 7).

According to the experience gained during the first run of the LHC, the aperture of the existing triplet may still be compatible with a $\beta^{*}$ of $40 \mathrm{~cm}$ in collision [30], which is already sensibly smaller than the design value of $55 \mathrm{~cm}$ [1]. Therefore the $40 \mathrm{~cm}$ ATS presqueezed optics is presently considered as a very good candidate for running the LHC after its restart in 2015. Also, a careful validation and detailed measurements of this optics [27] have already been carried out during the ATS beam experiments which were conducted in 2011 and 2012. The telescopic part of the squeeze is then described below starting from the presqueezed optics corresponding to case 1 in Table I, i.e. with $\beta_{\text {presqueeze }}^{*}=40 \mathrm{~cm}$.

\section{The telescopic collision optics}

The matching procedure of the telescopic squeeze has already been described in Sec. II B 2. Additional optics constraints, more specific to the left and right supporting insertions, shall also be taken into account during this process.
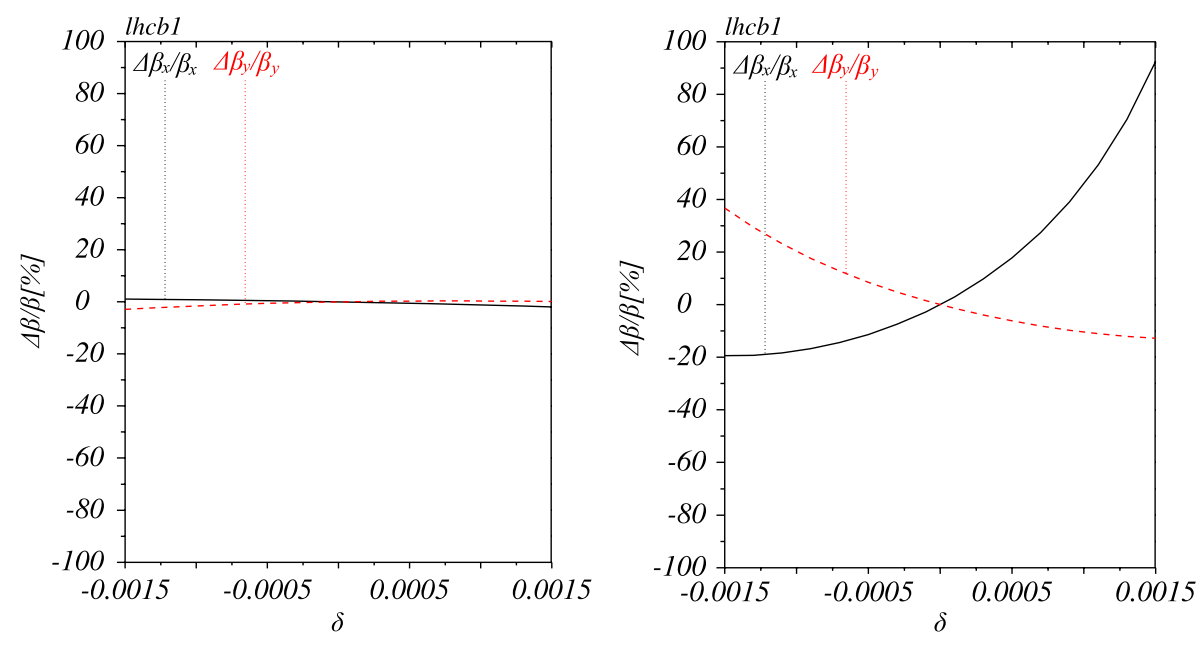

(a): $\frac{\Delta \beta(\delta)}{\beta}[\%]$ at IP5 (ATS)

(b): $\frac{\Delta \beta(\delta)}{\beta}[\%]$ at IP5 (nominal-like)
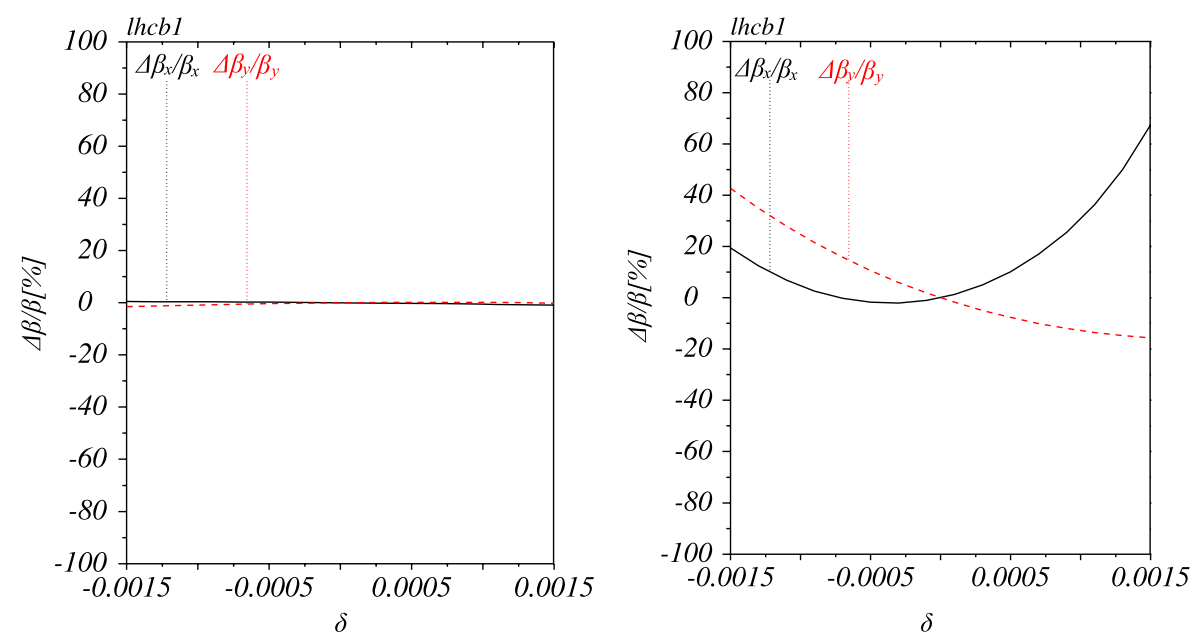

(c): $\frac{\Delta \beta(\delta)}{\beta}[\%]$ at IP7 (ATS)

(d): $\frac{\Delta \beta(\delta)}{\beta}[\%]$ at IP7 (nominal-like)

FIG. 7. Chromatic variations of the $\beta$ functions [\%] at IP5 (top) and IP7 (bottom), shown on the left for an ATS optics presqueezed down to $\beta^{*}=36 \mathrm{~cm}$ at IP1 and IP5 (see case 2 of Table I), and compared to a nominal-like LHC collision optics with $\beta^{*}=35 \mathrm{~cm}$ (right pictures). 
A first example concerns the Twiss parameters at IP4 which are kept constant during the telescopic squeeze, in order to minimize the variations of the $\beta$ functions in the core of the IR4 insertion which houses the rf equipments and most of the beam diagnostic systems. A second example is related to the choice of $\beta^{*}$ at the IPs of the two low-luminosity insertions IR2 and IR8. In the context of the ATS, these two IRs

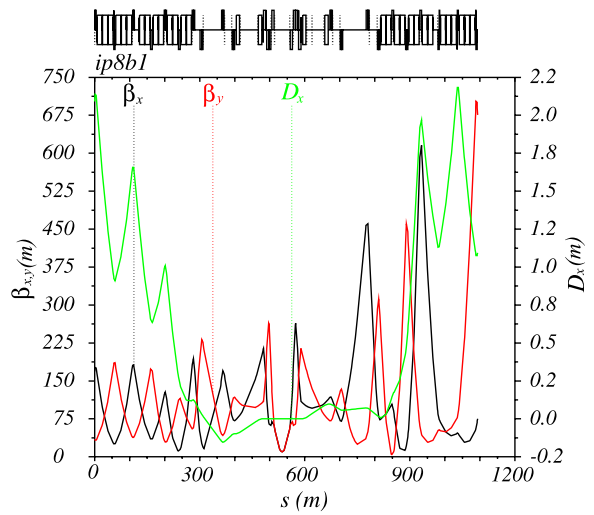

(a): $\operatorname{IR} 8\left(\beta^{*}=10 \mathrm{~m}\right)$

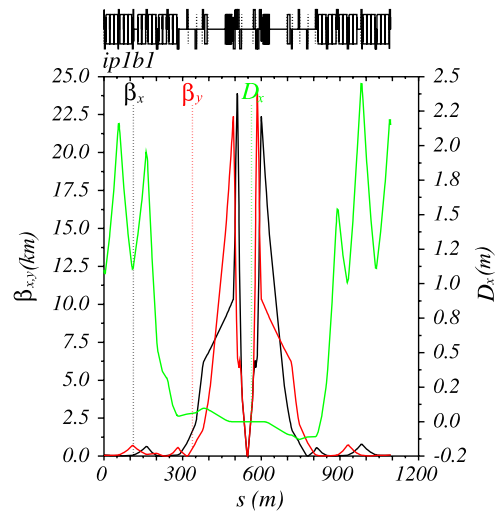

(b): $\operatorname{IR} 1\left(\beta_{x, y}^{*}=10 / 10 \mathrm{~cm}\right)$

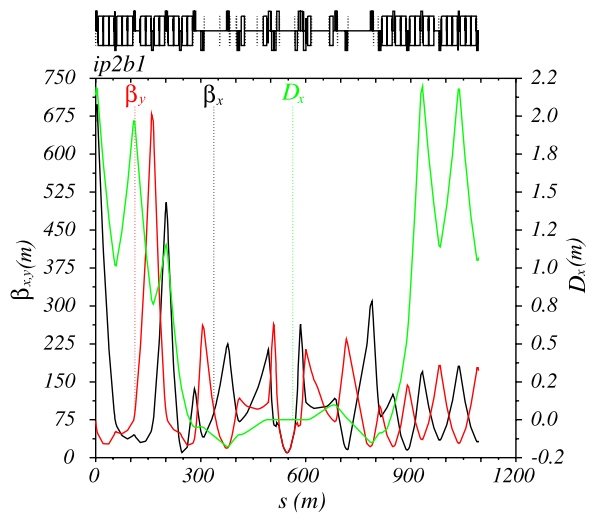

(c): $\operatorname{IR2}\left(\beta^{*}=10 \mathrm{~m}\right)$ shall indeed be seen as the left and right supporting insertions for the ATLAS experiment (see Fig. 1).

In the two examples shown in Fig. $8, \beta^{*}$ has been kept equal to its injection value of $10 \mathrm{~m}$ at IP2 and IP8, while a $\beta^{*}$ of $2-3 \mathrm{~m}$ is in principle within reach at these two IPs, without impacting on the telescopic squeeze of IR1 (see e.g. [31]). Starting from a presqueezed optics matched

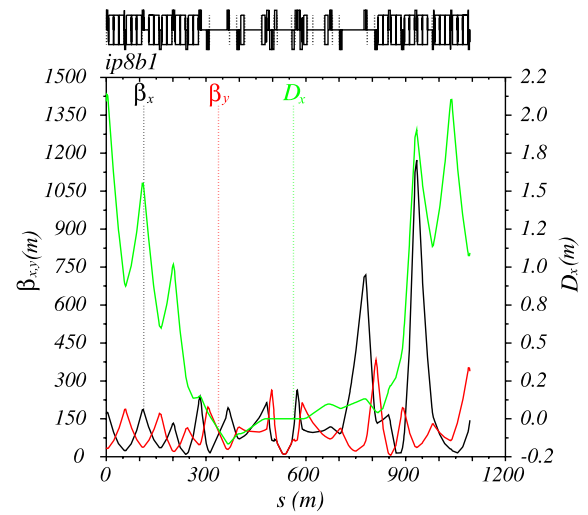

(d): $\operatorname{IR} 8\left(\beta^{*}=10 \mathrm{~m}\right)$

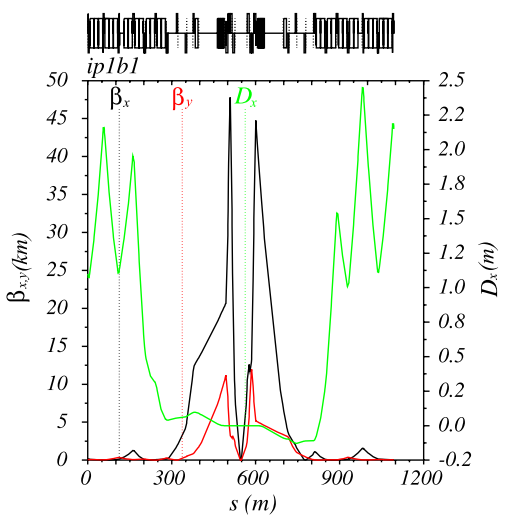

(e): $\operatorname{IR} 1\left(\beta_{x, y}^{*}=5 / 20 \mathrm{~cm}\right)$

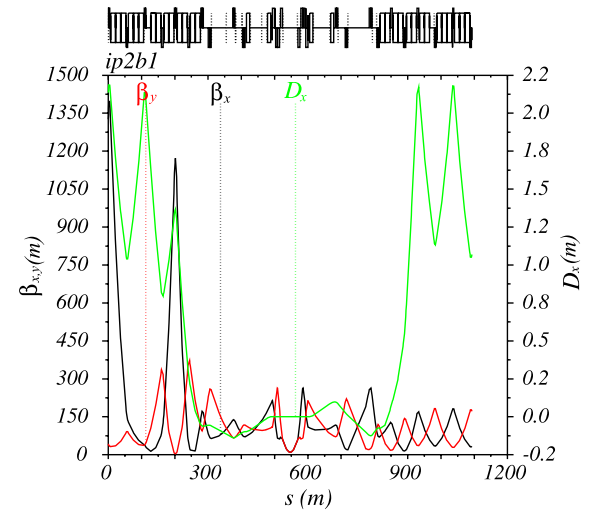

(f): $\operatorname{IR} 2\left(\beta^{*}=10 \mathrm{~m}\right)$

FIG. 8. Round (left pictures) and flat (right pictures) telescopic optics zoomed in IR8, IR1, and IR2, with $\beta_{x, y}^{*}=10 / 10 \mathrm{~cm}$ and $\beta_{x, y}^{*}=5 / 20 \mathrm{~cm}$ at IP1, respectively. The peak $\beta$ functions reached in the triplets of IR1 amounts to about $25 \mathrm{~km}$ in the first case (b), and up to $50 \mathrm{~km}$ in the second case (e). All these optics are shown with the crossing scheme switched off in IR1, IR2, IR5, and IR8 in order to preserve the dispersion matching (see also Sec. III C). 
to $\beta_{\text {presqueeze }}^{*} \equiv 40 \mathrm{~cm}$ [see Fig. 5(a)], the matching quadrupoles of IR2, IR8, IR4, and IR6 are then used in order to gain an additional squeeze factor from 4 to 8 , leading to a round telescopic optics with $\beta_{x, y}^{*}=10 \mathrm{~cm}$ in both planes

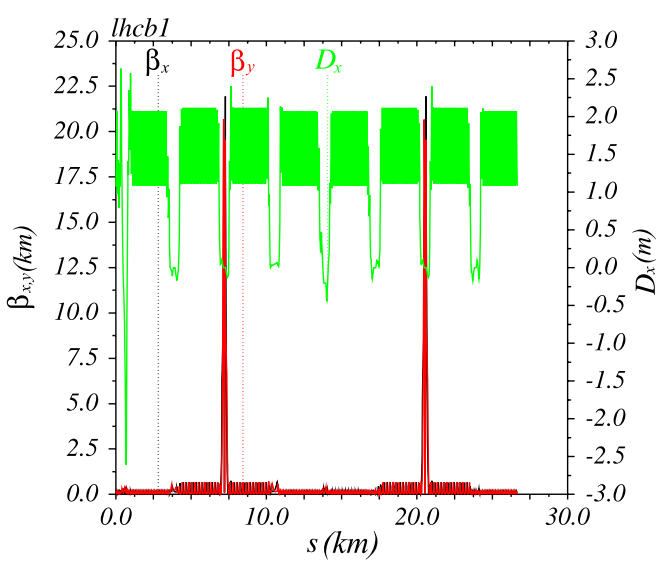

(a): Round ATS optics

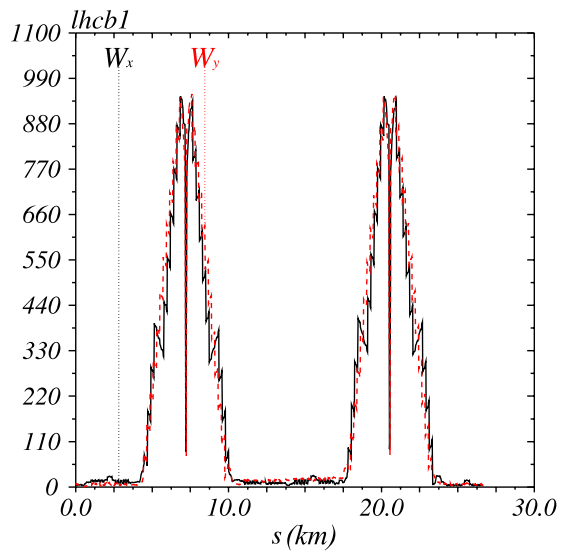

(c): $W_{x, y}(s)$ (round ATS optics)

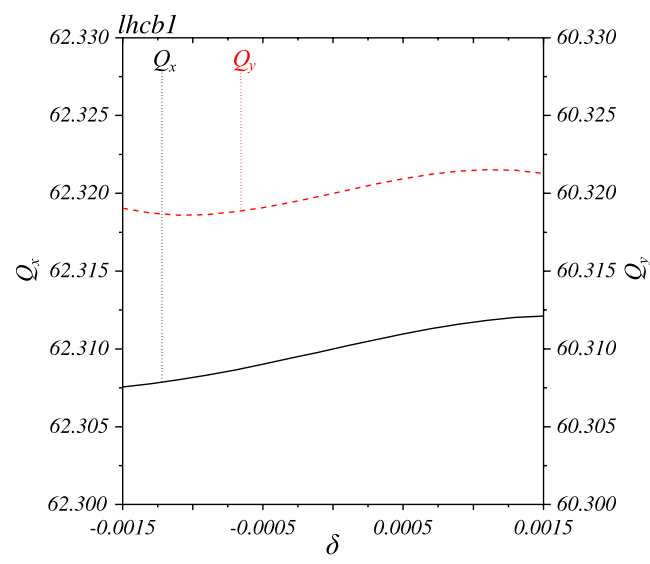

(e): $Q_{x, y}(\delta)$ (round ATS optics) at IP1 and IP5, or to a flat optics with $\beta_{x, y}^{*}=5 / 20 \mathrm{~cm}$ at IP1, and conversely at IP5 $\left(\beta_{x, y}^{*}=20 / 5 \mathrm{~cm}\right)$. In the case of IP1, and keeping $\beta^{*}$ constant at IP2 and IP8, a $\beta$-beating wave is initiated in arc 81 , as clearly visible on the right

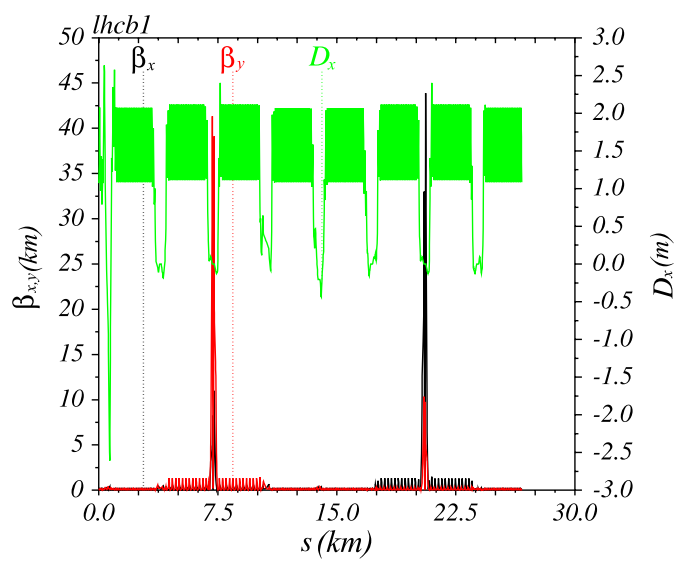

(b): Flat ATS optics

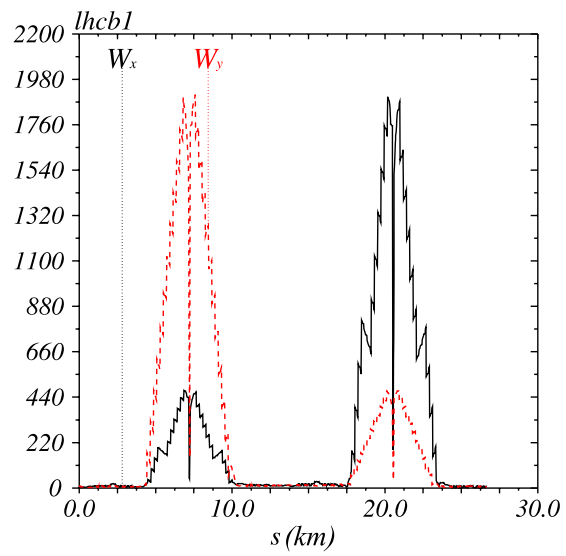

(d): $W_{x, y}(s)$ (flat ATS optics)

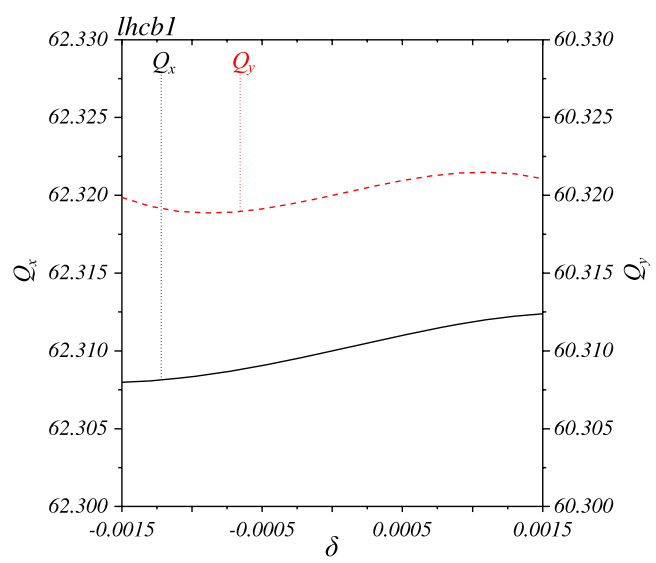

(f): $Q_{x, y}(\delta)$ (flat ATS optics)

FIG. 9. Typical round (left pictures) or flat (right pictures) telescopic optics for the HL-LHC, with $\beta_{x, y}^{*}=10 \mathrm{~cm}$ in both planes at IP1 and IP5, or $\beta_{x, y}^{*}=5 / 20 \mathrm{~cm}$ (respectively $20 / 5 \mathrm{~cm}$ ) at IP1 (respectively IP5). The machine is cycled from IP3: comparison in terms of $\beta$ and dispersion functions [(a) and (b)], Montague functions [(c) and (d)], and chromatic variations of the betatron tunes [(e) and (f)]. The crossing scheme has been switched off in the four experimental IRs in order to preserve the dispersion matching (see also Sec. III C). 
side of IR8 [see Figs. 8(a) and 8(d)], which contributes to the telescopic squeeze of IR1, before being closed thanks to the matching quadrupoles of IR2 [see Figs. 8(c) and 8(f)]. In this process, the horizontal and vertical betatron phase advances from Q13.L8 to IP1 and from IP1 to Q13.R2 are also kept unchanged with respect to the presqueezed optics in order to preserve the betatron tunes.

The corresponding overall optics are showed in Figs. 9(a) and 9(b) together with the associated Montague functions $W_{x, y}$ [Figs. 9(c) and 9(d)], and the chromatic variations of the betatron tunes [Figs. 9(e) and 9(f)]. A zoom of these two optics in arc 45 was already given in Figs. 4(b) and 4(c). Even for the flat optics with a $\beta^{*}$ as small as $5 \mathrm{~cm}$ in one of the two transverse planes, and a corresponding $\beta_{\max }$ of almost $50 \mathrm{~km}$ reached in the inner triplets of IR1 and IR5, the $W$ functions remain perfectly under control, and a very modest third order chromaticity $Q^{\prime \prime \prime}$ starts to show up. These results can be directly compared with the situation shown in Figs. 6(b) and 6(d) for a nominal-like LHC collision optics where $\beta^{*}$ was still almost 1 order of magnitude higher.

In order to reach such a quality for the chromatic correction, in particular for the nonlinear chromaticity, a finetuning of the left and right phase advances of the low- $\beta$ insertions is needed, as explained in the end of the Sec. II B 3. This final numerical optimization is first performed using the two telescopic optics mentioned above, and is then used to rephase accordingly the presqueezed optics, and finally the injection optics. One single iteration is generally sufficient, back and forth between the telescopic and the presqueezed optics, in order to reach a very good quality for the chromatic correction. For the extreme $\beta^{*}$ values of $5-10 \mathrm{~cm}$ under consideration, the chromatic correction is sensitive to variations of the order of $0.5-1$ degrees $\left(2-3 \times 10^{-3}\right.$ in tune units) for the left and right phase advances of the low- $\beta$ insertions. This requirement is challenging, but actually not far from what has been already demonstrated in the LHC where the $\beta$-beating correction is actually based on the measurement of the betatron phase advances and on the minimization of their deviation with respect to the model [32].

Finally, as for the presqueezed optics, and both for the round and flat telescopic optics under consideration, the chromatic variations of the $\beta$ functions do not exceed the percent level in the collimation insertions IR3 and IR7 [see Figs. 10(c) and 10(d) for IP7]. The situation is qualitatively and quantitatively different when inspecting these variations at the interaction points of the high luminosity insertions, as illustrated in Figs. 10(a) and 10(b) in the case of IP5. A first order off-momentum $\beta$ beating $\partial_{\delta} \beta$ shows up, which comes from the final optimization of the chromatic correction in terms of second order chromaticity $Q^{\prime \prime}$. On the other hand, this term alone does only contribute to a very small fraction of the total chromatic variations of the $\beta$ functions at the IP. The main contribution comes from a second order off-momentum $\beta$ beating $\partial_{\delta}^{2} \beta$. The latter is always positive at the IP and therefore negative in the final focus quadrupoles, which means that it does not degrade the off-momentum aperture of the inner triplet. The existence of this term is intrinsic to the strategy which has been adopted for the correction of the $W$ Montague function. More precisely, the off-momentum $\alpha$ beating is not strictly vanishing at the IP, and given by

$$
\alpha_{x, y}^{*}(\delta) \sim \Delta I_{x, y} \delta,
$$

where the quantities $\Delta I_{x, y}$ are defined in Eq. (6). Then, since the Twiss parameters are linked by the usual relation $\beta \gamma=1+\alpha^{2}$ for any momentum errors, this residual offmomentum $\alpha^{*}$ contributes as follows to the second order off-momentum $\beta$ beating at the IP:

$$
\left(\frac{\partial^{2} \beta_{x, y}^{*}}{\partial \delta^{2}}\right)_{(\delta=0)} \sim 2 \frac{\left(\Delta I_{x, y}\right)^{2}}{\gamma_{x, y}^{*}(0)} \propto \frac{\left(\Delta I_{\text {prequeeze }}\right)^{2}}{\beta_{x, y}^{*}},
$$

which would be vanishing for a symmetric optics, but is increasing as $1 / \beta^{*}$ during the telescopic squeeze of the LHC antisymmetric optics. On the other hand, with a rms energy spread of $1-2 \times 10^{-4}$ for the (HL)-LHC beam at high energy [1,2], this term has still a very marginal impact onto the performance of the machine, and remains really small compared to nominal collision optics of much larger $\beta^{*}$.

\section{Spurious dispersion from the crossing angle and its efficient correction for ATS optics}

Contrary to other existing colliders like RHIC [33], the LHC has this particularity that the beams collide with a nonzero crossing angle at the IP. For reasons related to beam-beam effects, the crossing planes are alternated in the two high luminosity insertions of the machine, with presently a vertical crossing angle in IR1, and horizontal in IR5 (see e.g. [34]). When reducing $\beta^{*}$, the crossing angle shall also be increased with $1 / \sqrt{\beta^{*}}$ in order to keep constant the normalized separation between the two beams at the long-range (LR) beam-beam encounters where the beams still interact with each other. The LHC counts 15 LR encounters on either side of IP1 or IP5, until reaching the separation dipole D1 located on the non-IP side of the triplet (see Fig. 2). This number is typically increased to about 20 for triplet quadrupoles of larger aperture, therefore operating at lower gradient, and then being longer in proportion compared to the existing low- $\beta$ quadrupoles of the LHC. For the HL-LHC, the minimum normalized separation at the long-range encounters has been estimated to be about $d_{\mathrm{bb}} \sim 12.5 \sigma$ for round telescopic optics, corresponding to a half-crossing angle of

$$
\Theta_{c} \equiv \frac{d_{\mathrm{bb}}}{2} \times \sqrt{\frac{\epsilon}{\beta^{*}}}
$$

that is $\Theta_{c}=360 \mu \mathrm{rad}$ for $\beta^{*}=10 \mathrm{~cm}$ [23], at an energy of $7 \mathrm{TeV} /$ beam and assuming a normalized emittance of $\gamma \epsilon=2.5 \mu \mathrm{m}$ for the HL-LHC beam [2]. With such a 


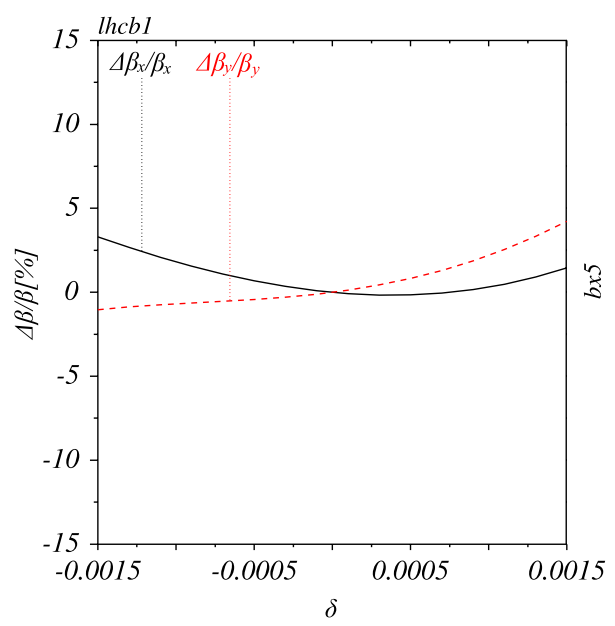

(a): $\frac{\Delta \beta(\delta)}{\beta}[\%]$ at IP5 (round ATS)

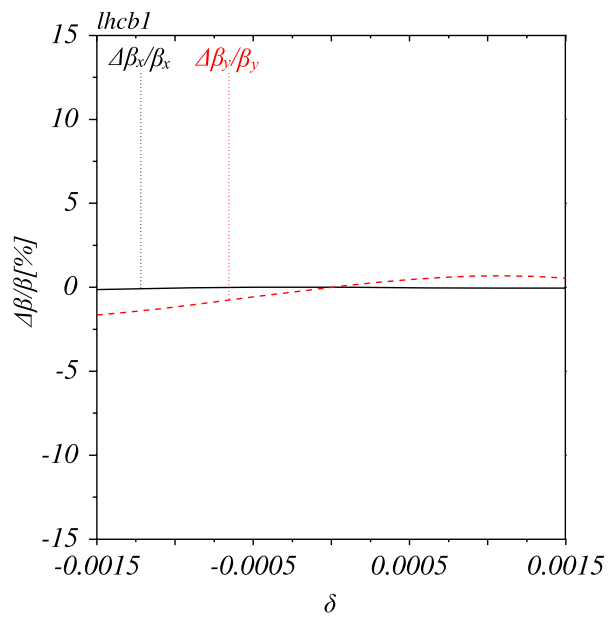

(c): $\frac{\Delta \beta(\delta)}{\beta}[\%]$ at IP7 (round ATS)

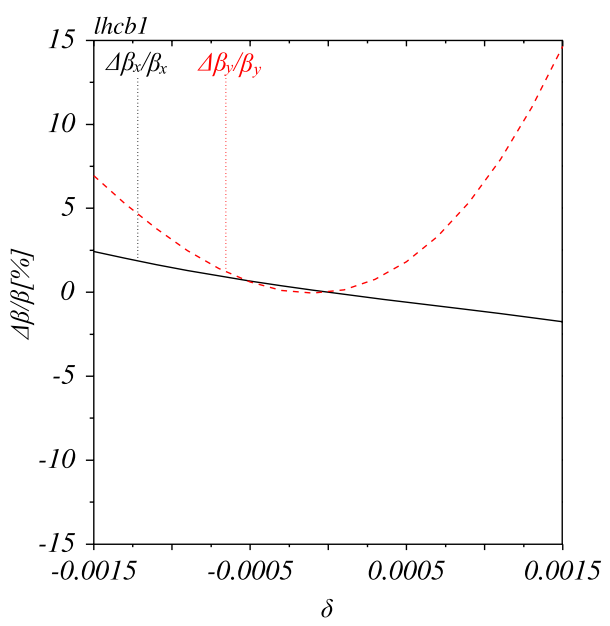

(b): $\frac{\Delta \beta(\delta)}{\beta}[\%]$ at IP5 (flat ATS)

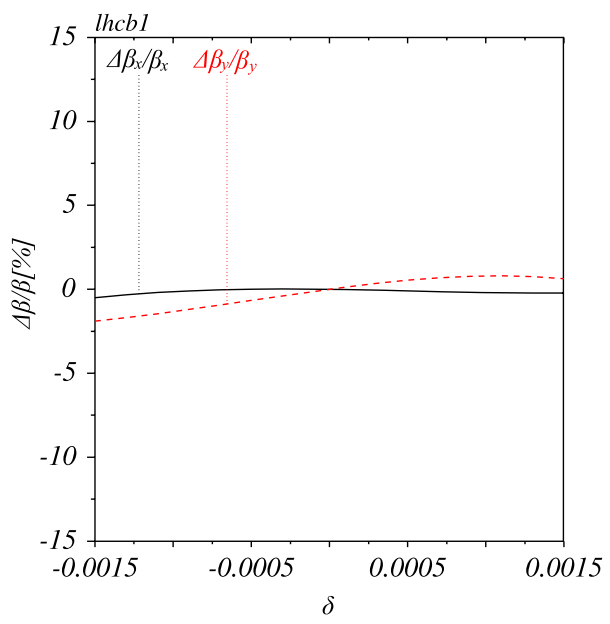

(d): $\frac{\Delta \beta(\delta)}{\beta}[\%]$ at IP7 (flat ATS)

FIG. 10. Chromatic variations of the $\beta$ functions [\%] at IP5 (top) and IP7 (bottom) for the telescopic round (left) and flat (right) optics considered in Fig. 9.

crossing angle, the peak horizontal or vertical beam excursion, namely $\Delta \hat{z}_{\mathrm{TT}}$, is as high as $15 \mathrm{~mm}$ in the inner triplets of IR1 and IR5 [see the two highest peaks of closed orbit in Fig. 11(a)]. This in turn induces a wave of horizontal and vertical spurious dispersion all around the ring, which can reach up to 10-20 $\mathrm{m}$ in the final focus quadrupoles of the high luminosity insertions [see Fig. 11(b)], to be compared for instance with a nominal horizontal dispersion of maximum $2 \mathrm{~m}$ in the arcs. The spurious dispersion induced by the crossing angle in one of the two high luminosity insertions of the LHC, and then exported in the inner triplets of the second IR, depends obviously on the betatron phase advances between these two insertions. For alternated crossing scheme, however, the horizontal and vertical spurious dispersions cannot be vanishing simultaneously in the two high luminosity insertions, unless an active correction is deployed.

Indeed, without corrective action, the following scaling law is expected for the normalized amplitude of the spurious dispersion wave induced by the crossing angle in a given low- $\beta$ insertion:

$$
\left|\frac{\Delta D(s)}{\sqrt{\beta(s)}}\right| \propto \Delta \hat{z}_{\mathrm{IT}} \sqrt{\hat{\beta}_{\mathrm{IT}}} \sim \sqrt{\beta^{*}} \hat{\beta}_{\mathrm{IT}} \times \Theta_{c} \propto \frac{1}{\sqrt{G}} \frac{d_{\mathrm{bb}}}{\beta^{*}},
$$

where the relations (1) and (37) have been used. Therefore, reducing the gradient $G$ to maximize the triplet aperture makes again the situation slightly worse in terms of optics. While this spurious dispersion is of the order of $1-2 \mathrm{~m}$ for the nominal collision optics of the LHC $\left(\beta^{*}=55 \mathrm{~cm}\right)$, it can then be 1 order of magnitude higher for the HL-LHC, and therefore have very detrimental effects in terms of performance: reducing the mechanical acceptance of the inner triplet (and therefore the minimum possible $\beta^{*}$ ), contributing to an increase of the effective spot sizes at the IP, but also leading to more subtle effects as a shift of the linear chromaticity in the end of the squeeze induced by the sextupole-like component of the long-range 


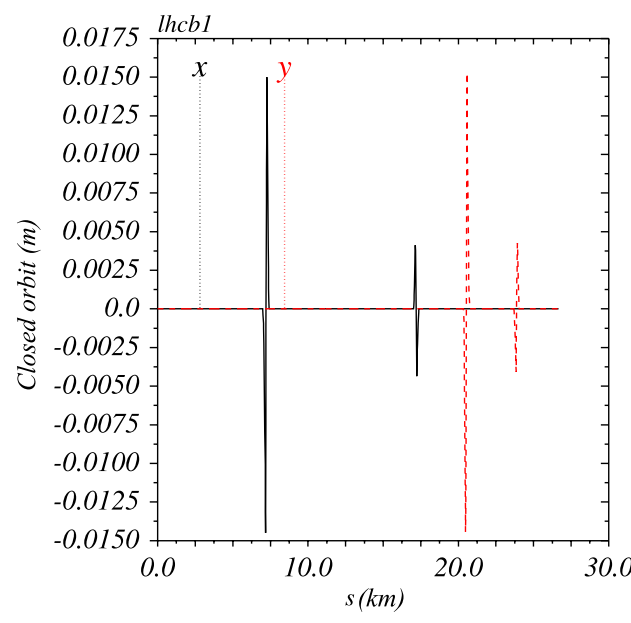

(a): $\mathrm{H}$ and $\mathrm{V}$ closed orbit $[\mathrm{m}]$

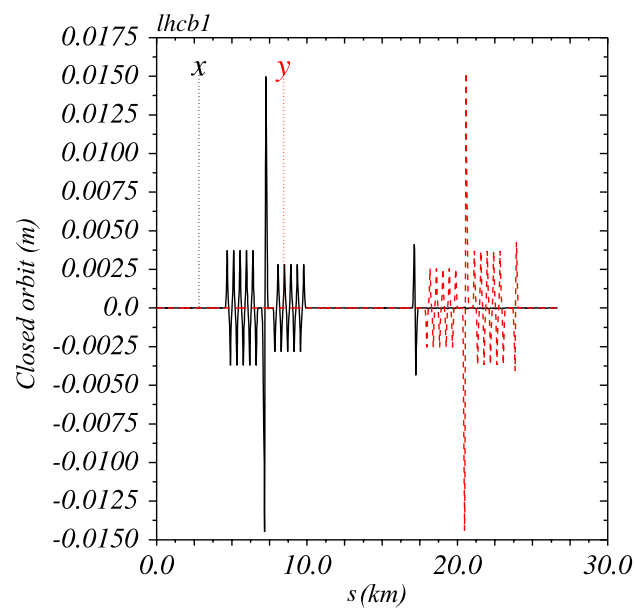

(c): H and V closed orbit [m]

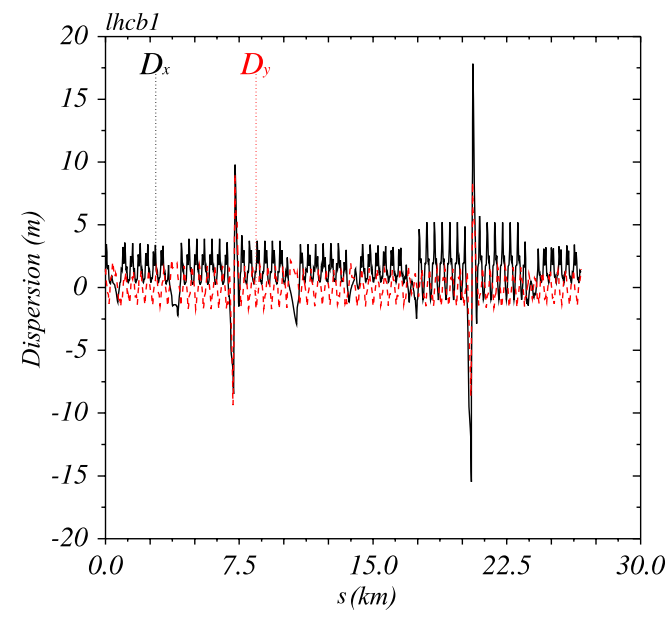

(b): $\mathrm{H}$ and $\mathrm{V}$ dispersion $[\mathrm{m}]$

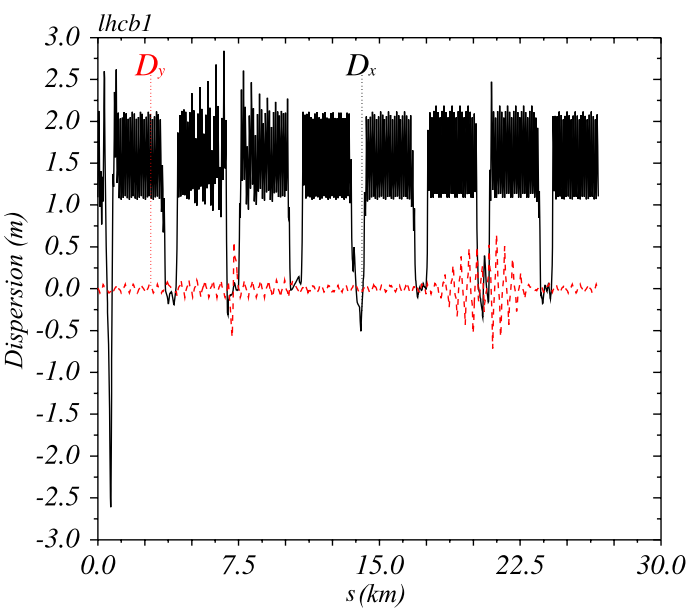

(d): $\mathrm{H}$ and $\mathrm{V}$ dispersion $[\mathrm{m}]$

FIG. 11. Horizontal and vertical closed orbit (left pictures) and dispersion (right pictures) around the LHC ring for the round ATS optics considered in Fig. 9(a) with $\beta^{*}=10 \mathrm{~cm}$ and a crossing angle of $720 \mu \mathrm{rad}$. The machine is cycled from IP3 for the clockwise rotating Beam1. The highest orbit excursions shown in (a) result from the crossing angles at IP5 (horizontal) and IP1 (vertical), while the smaller peaks on either side of the second highest peak are due to the H and V crossing angles at IP8 and IP2, respectively. The dispersion is fully mismatched in the first case (top pictures). In the second case (bottom picture), horizontal (respectively vertical) orbit bumps are generated in the arcs 45 and 56 (respectively 81 and 12), reducing the dispersion mismatch by more than 1 order of magnitude [note the change of vertical scale between (b) and (d)].

beam-beam interaction, and leading as well to variations by up to $\Delta Q_{\mathrm{bb}}^{\prime} \sim \pm 10$ units along the HL-LHC bunch trains since different bunches do not necessarily experience the same number of LR beam-beam encounters.

The problem could in principle be solved in the horizontal plane, without the ATS scheme, by keeping the crossing angle switched on when matching the collision optics of a given low- $\beta$ insertion. This approach is however relatively costly in terms of optics flexibility, and therefore minimum possible $\beta^{*}$. It is in any case not suitable for the ATLAS experimental insertion where the crossing angle is vertical, and therefore is not used in practice. Actually, the LHC was (a priori) not designed to control properly the spurious dispersion in the vertical plane, at least not a spurious dispersion of the magnitude expected for the HL-LHC. Indeed, a method of correction tried out in the past was to generate vertical (or horizontal) orbit bumps in the LHC arcs [35]. This analysis performed on the nominal collision optics demonstrated that this strategy could work, but leading to a peak closed orbit of up to $\pm 6.5 \mathrm{~mm}$ in the arcs. This would mean at least 5 times more for the low $\beta^{*}$ targeted for the HL-LHC [see Eq. (38)], and therefore well beyond the mechanical acceptance of the arcs (and capability of the orbit correctors to generate the bumps at flattop energy). In fact, this strategy does work when combined with the ATS scheme, essentially thanks to the phasing properties of the presqueezed optics. More quantitatively, assuming that 
relatively modest horizontal (respectively vertical) orbit bumps, not exceeding $\pm 4 \mathrm{~mm}$ peak to peak, are generated in the two arcs on either of IP5 (respectively IP1) where the crossing angle is horizontal (respectively vertical), the horizontal (respectively vertical) dispersion can be very well controlled all around the ring [see Figs. 11(c) and 11(d)]. In particular, the negative dispersion needed in IR3 for momentum collimation is fully restored as shown on the left side of Fig. 11(d), the spurious dispersion does not exceed $50 \mathrm{~cm}$ in the inner triplets of IR1 and IR5 (which means less than $1 \mathrm{~mm}$ at the IP), and remains as well very small in the betatron collimation insertion IR7. This correction leaves however a small residual due to the crossing angles in IR2 (Alice experiment) and IR8 (LHCb).

The fundamental reason why ATS optics are very efficient for the correction of the spurious dispersion via orbit bumps is a direct consequence of their intrinsic properties, in terms of betatron phase advances, chromatic correction, and techniques used for the telescopic squeeze (see Secs. II B 1 and II B 2, respectively). The detailed analysis is reported in Appendix B, leading in particular to a very simple expression for the normalized amplitude of the orbit bumps which is needed in the two arcs on either side of the IP:

$$
d_{\mathrm{co}} \sim \frac{d_{\mathrm{bb}}}{2} .
$$

For a minimum required beam-beam separation of $d_{\mathrm{bb}}=$ $12.5 \sigma$, the above relation leads to a peak closed orbit of about $2 \mathrm{~mm}$ in the arcs for a typical (HL)-LHC presqueezed optics with $\beta^{*}=40-50 \mathrm{~cm}$, and only $4 \mathrm{~mm}$ for typical HL-LHC telescopic optics, where the peak $\beta$ functions would be increased by a factor of 4 in the arcs in order to further reduce $\beta^{*}$ in proportion at the IP.

Finally, to first order in the amplitude of the orbit bumps in the arcs, the above correction strategy has no impact on tune shift, $\beta$ beating, and linear coupling, since the normal or skew quadrupole field errors induced by feed-down effects in the strong sextupoles change of sign from magnet to magnet. Indeed the strong sextupoles are spaced by $\pi$ in betatron phase, and each family contains an even number of magnets. Furthermore, this strategy is also orthogonal to the chromatic correction (linear and nonlinear chromaticity, off-momentum $\beta$ beating), since the dispersion wave induced by the strong sextupoles is out of phase by $\pi / 2$ with respect to the peak $\beta$ functions reached in the arcs, which also means that it is vanishing at the strong sextupoles.

\section{SUMMARY AND CONCLUSIONS}

The achromatic telescopic squeezing scheme represents a real evolution with respect to the standard concept of a low- $\beta$ insertion, where the reduction of $\beta^{*}$ is generally achieved by using a certain number of matching quadrupoles, confined in a rather small fraction of the ring on either side of the IP. In order to overcome a series of optics limitations which are driven at low $\beta^{*}$ either by the gradient limits imposed to the matching quadrupoles or by the strength available in the lattice sextupoles, the ATS scheme extends the physical limits of the low- $\beta$ insertion (from the optics point of view), and splits the new corresponding insertion into three well-identified blocks: (1) two supporting insertions playing the role of left and right matching sections (located several $\mathrm{km}$ upstream and downstream of the ATLAS and CMS experimental insertions in the case of the LHC); (2) two arcs of machines on either side of the IP playing the role of two interleaved horizontal and vertical chromatic correction sections, for compensating the chromatic aberrations and eventually the spurious dispersion induced by the final focus quadrupoles; (3) and the low- $\beta$ insertion proper, playing the role of a final telescope which becomes strictly passive below a certain $\beta^{*}$ (the presqueezed $\beta^{*}$ ). The reduction of $\beta^{*}$, basically without any chromatic limits, and a proper chromatic correction are intimately connected in the ATS scheme. This makes this scheme already very attractive for the existing LHC machine, bringing in particular a definite solution for the long-standing problem of correcting the off-momentum $\beta$ beating, but also the horizontal or vertical spurious dispersion induced by the crossing angle in the four experimental insertions of the ring. While magnets of larger aperture are obviously a prerequisite for a further reduction of $\beta^{*}$, this condition combined with the ATS scheme is not only necessary but becomes also sufficient to reach very low $\beta^{*}$. With its universality versus triplet layout and technology, its flexibility, in particular to deliver round or flat collision optics at constant layout, and mainly its unprecedented potential $\beta^{*}$ reach thanks to an optimal usage of the entire ring in terms of matching quadrupoles, sextupoles, but also aperture available in the arcs at flattop energy, the ATS scheme opens the path towards the performance targeted by the HL-LHC project. Consequently, it certainly strongly influenced the hardware direction which was taken by the project, in particular with magnets of the largest possible aperture, and became rapidly a vital ingredient for the luminosity upgrade program of the LHC.

\section{ACKNOWLEDGMENTS}

I am sincerely grateful to S. Myers who strongly supported the ATS scheme as soon as I brought it to his attention. I would also like to thank R. de Maria who helped me to analyze rapidly its main potential limitations, in particular its negative impact onto the dynamic aperture which was hopefully found more than acceptable for the LHC. This was certainly a strong motivation in the early development of the ATS scheme. Although its first validation with beam will form the content of another publication, the very successful results obtained at the LHC gave certainly a non-negligible momentum to the ATS. In this context, I can only strongly acknowledge the precious help and constant support that I received from the LHC optics measurement and correction team, and in particular from 
its leader R. Tomas, and of course from the LHC operation team, in particular M. Lamont, G. Mueller, L. Ponce, S. Redaelli, J. Wenninger, and many others. Finally I would like to express my gratitude to M. Giovannozzi and the two referees for the accurate and patient work of proofreading and commenting the draft manuscript of this article. The documentation of this work was supported by FP7 HiLumi LHC [36] (Grant Agreement No. 284404).

\section{APPENDIX A: FINE-TUNING OF THE SEXTUPOLE SETTINGS DURING THE TELESCOPIC SQUEEZE AND RESIDUAL CHROMATICITY}

This Appendix will analyze in more detail the evolution of the arc optics during the telescopic squeeze, the finetuning of the strong sextupoles which is needed for a precise control of the off-momentum $\beta$ beating during this process, and the residual linear chromaticity which is left uncorrected and shall be compensated by the other sextupoles of the lattice. Most of the notations used below have been already defined in the Secs. II B 1 and II B 2.

Since $\alpha^{*}$ is kept equal to zero, the following relation holds during the telescopic part of the squeeze:

$$
\sqrt{\tilde{\beta}(s)} e^{j \Delta \tilde{\mu}(s)} \equiv \sqrt{\tilde{\beta}^{*}} R_{22}(s)+j / \sqrt{\tilde{\beta}^{*}} R_{12}(s),
$$

where $\tilde{\beta}(s)$ is the new (mismatched) $\beta$ function at a given position $s$ in between the left and right supporting insertions, $\Delta \tilde{\mu}(s)$ the new phase advance from this position to the IP, and $R_{12,22}(s)$ are the $\left(x, x^{\prime}\right)$ and $\left(x^{\prime}, x^{\prime}\right)$ coefficients of the corresponding $R$ matrix. The quadrupole settings being kept constant both in the low- $\beta$ insertion and in the neighboring arcs, this $R$ matrix is invariant during the telescopic squeeze. Therefore, its coefficients can also be described by the unperturbed $\beta$ function $\beta(s)$ and phase advance $\Delta \mu(s)$ of the presqueezed optics:

$R_{12}(s)=\sqrt{\beta(s) \beta_{0}^{*}} \sin [\Delta \mu(s)], \quad R_{22}(s)=\sqrt{\frac{\beta(s)}{\beta_{0}^{*}}} \cos [\Delta \mu(s)]$.

Combining the two above relations, and after some algebra, one gets

$$
\begin{aligned}
\tilde{\beta}(s)= & \frac{1}{2} \beta(s)[\{1-\cos [2 \Delta \mu(s)]\} \\
& \left.+\{1+\cos [2 \Delta \mu(s)]\}\left(\frac{\tilde{\beta}^{*}}{\beta_{0}^{*}}\right)^{2}\right]\left(\frac{\beta_{0}^{*}}{\tilde{\beta}^{*}}\right),
\end{aligned}
$$

and

$$
\begin{aligned}
\tilde{\beta}(s) e^{2 j \Delta \tilde{\mu}(s)=} & \frac{1}{2} \beta(s)\left[\cos [2 \Delta \mu(s)]\left(\frac{\tilde{\beta}^{*}}{\beta_{0}^{*}}+\frac{\beta_{0}^{*}}{\tilde{\beta}^{*}}\right)\right. \\
& \left.+\left(\frac{\tilde{\beta}^{*}}{\beta_{0}^{*}}-\frac{\beta_{0}^{*}}{\tilde{\beta}^{*}}\right)\right]+j \beta(s) \sin [2 \Delta \mu(s)] .
\end{aligned}
$$

The imaginary part of the phasor $\tilde{\beta} e^{2 j \Delta \tilde{\mu}}$ is therefore invariant during the telescopic squeeze, contrary to the real part which depends on the new $\beta^{*}$ value, namely $\tilde{\beta}^{*}$. This simple fact has a direct consequence on the $(2,0)$ and $(0,2)$ chromatic driving terms which are generated by the strong sextupoles. Indeed these driving terms will be kept in phase with respect to the final focus quadrupoles if and only if the new settings of the strong sextupoles remains collinear to the ones previously established for the presqueezed optics [see Eq. (19)]:

$$
\begin{aligned}
& \Delta \tilde{K}_{2_{F}}^{L, R}=\frac{\lambda}{N_{F}^{L, R} \beta_{F} D_{x_{F}}} \times \frac{I_{0}}{\sqrt{1+r_{F D}^{2}}}, \\
& \Delta \tilde{K}_{2_{D}}^{L, R}=-\frac{\lambda}{N_{D}^{L, R} \beta_{F} D_{x_{D}}} \times \frac{I_{0}}{\sqrt{1+r_{F D}^{2}}},
\end{aligned}
$$

where all the quantities in the rhs refer to the unperturbed (presqueezed) optics, and the multiplicative factor $\lambda$ depends on the new $\beta^{*}$. In order to determine the $\lambda$ factor, the achromaticity conditions (11) and (12) need to be rewritten for the perturbed optics,

$$
\begin{aligned}
& N_{F}^{L, R} \tilde{\beta}_{F_{x}} D_{x_{F}} \Delta \tilde{K}_{2_{F}}^{L, R}+N_{D}^{L, R} \tilde{\beta}_{D_{x}} D_{x_{D}} \Delta \tilde{K}_{2_{D}}^{L, R} \equiv \tilde{I}_{x}, \\
& N_{F}^{L, R} \tilde{\beta}_{F_{y}} D_{x_{F}} \Delta \tilde{K}_{2_{F}}^{L, R}+N_{D}^{L, R} \tilde{\beta}_{D_{y}} D_{x_{D}} \Delta \tilde{K}_{2_{D}}^{L, R} \equiv-\tilde{I}_{y},
\end{aligned}
$$

and

$$
\begin{aligned}
& e^{2 j\left(\Delta \tilde{\mu}_{x}^{L, R}-\pi / 2\right)}\left[N_{F}^{L, R} \tilde{\beta}_{F_{x}} D_{x_{F}} \Delta \tilde{K}_{2_{F}}^{L, R}\right. \\
& \left.\quad+e^{2 j \Delta \tilde{\mu}_{x_{F D}}^{L, R}} N_{D}^{L, R} \tilde{\beta}_{D_{x}} D_{x_{D}} \Delta \tilde{K}_{2_{D}}^{L, R}\right] \equiv \tilde{I}_{x}, \\
& e^{2 j\left(\Delta \tilde{\mu}_{y}^{L, R}-\pi / 2\right)}\left[e^{-2 j \Delta \tilde{\mu}_{y_{F D}}^{L, R}} N_{F}^{L, R} \tilde{\beta}_{F_{y}} D_{x_{F}} \Delta \tilde{K}_{2_{F}}^{L, R}\right. \\
& \left.\quad+N_{D}^{L, R} \tilde{\beta}_{D_{y}} D_{x_{D}} \Delta \tilde{K}_{2_{D}}^{L, R}\right] \equiv-\tilde{I}_{y},
\end{aligned}
$$

where the chromatic betatron kicks $\tilde{I}_{x, y}$ given by the inner triplet at the new $\tilde{\beta}^{*}$ can be deduced from the relation (4)

$$
\tilde{I}_{x, y} \equiv \frac{\beta_{0}^{*}}{\tilde{\beta}_{x, y}^{*}} \times I_{0} .
$$

At this stage the only parameter which is left free is the multiplicative constant $\lambda$ introduced in Eq. (A5). Therefore, a priori only one of the four previous conditions can be solved exactly. Considering one after the other the two conditions related to the cancellation of the $(2,0)$ and $(0,2)$ chromatic driving terms [Eq. (A7)], and using the relations (10), (13), (14), (20), (A4), and (A5) to determine the new contributions $\tilde{\beta} e^{2 j \Delta \tilde{\mu}} \Delta \tilde{K}_{2}$ of the strong sextupoles, one finds

$$
\lambda \sim \lambda_{x, y} \stackrel{\text { def }}{=} \frac{1}{1-\kappa\left[1-\left(\frac{\tilde{\beta}_{x, y}^{*}}{\beta_{0}^{*}}\right)^{2}\right]},
$$

with 


$$
\kappa \stackrel{\text { def }}{=} \frac{1}{2}\left[1-\frac{1-r_{F D}}{\sqrt{1+r_{F D}^{2}}}\right] .
$$

The relation (A9) implicitly tells that the $(2,0)$ and $(0,2)$ chromatic driving terms cannot be compensated simultaneously, except in the case of round telescopic optics $\left(\tilde{\beta}_{x}^{*} \equiv \tilde{\beta}_{y}^{*}\right)$, or asymptotically at extremely low $\beta^{*}$ $\left(\tilde{\beta}_{x, y}^{*} \ll \tilde{\beta}_{0}^{*}\right)$. On the other hand, for intermediate flat optics $\left(\tilde{\beta}_{x}^{*} \neq \tilde{\beta}_{y}^{*}\right)$ where the largest $\beta^{*}$ has already been reduced by a factor of 2 with respect to the presqueezed optics, it is easy to see that the $(2,0)$ and $(0,2)$ driving terms can both be corrected within a precision of $1 \%$ or better:

$$
\frac{\left|\lambda_{x}-\lambda_{y}\right|}{\lambda_{x}+\lambda_{y}} \approx \frac{\kappa}{2} \frac{\left|\left(\tilde{\beta}_{x}^{*}\right)^{2}-\left(\tilde{\beta}_{y}^{*}\right)^{2}\right|}{\left(\beta_{0}^{*}\right)^{2}} \lesssim \frac{\kappa}{8} \sim 1 \% .
$$

Finally, the coefficient $\kappa$, of the other of $r_{F D} / 2 \sim 10 \%$, gives the very modest variation range which is expected for the sextupole settings during the telescopic squeeze, regardless of the final collision $\tilde{\beta}^{*}$.

The locality of the chromaticity correction [see Eq. (A6)] is also greatly improved in comparison with the situation described in Sec. II B 1 for the presqueezed optics. Coming back to the relations (10), (13), (14), and (20) which characterize the presqueezed optics, and inserting these relations into (A3), the perturbed $\beta$ functions at the strong sextupoles can be written as follows:

$$
\begin{aligned}
& \tilde{\beta}_{F_{x}, D_{y}}=\beta_{F} \times\left[\lambda_{F}+\left(1-\lambda_{F}\right)\left(\frac{\tilde{\beta}_{x, y}^{*}}{\beta_{0}^{*}}\right)^{2}\right] \times\left(\frac{\beta_{0}^{*}}{\tilde{\beta}_{x, y}^{*}}\right), \\
& \tilde{\beta}_{D_{x}, F_{y}}=\beta_{D} \times\left[\lambda_{D}+\left(1-\lambda_{D}\right)\left(\frac{\tilde{\beta}_{x, y}^{*}}{\beta_{0}^{*}}\right)^{2}\right] \times\left(\frac{\beta_{0}^{*}}{\tilde{\beta}_{x, y}^{*}}\right),
\end{aligned}
$$

with

$$
\begin{aligned}
& \lambda_{F} \stackrel{\text { def }}{=} \frac{1}{2}\left[1+\frac{1}{\sqrt{1+r_{F D}^{2}}}\right] \approx 1, \\
& \lambda_{D} \stackrel{\text { def }}{=} \frac{1}{2}\left[1-\frac{r_{F D}}{\sqrt{1+r_{F D}^{2}}}\right] \approx \frac{1-r_{F D}}{2} .
\end{aligned}
$$

By inserting the above relations into the chromaticity correction condition (A6), and using Eqs. (A5) and (A9) which give the settings of the strong sextupoles during the telescopic squeeze [within the approximation (A11)], the residual uncorrected chromaticity is of the order of

$4 \pi \Delta Q_{x, y}^{\prime} \approx \frac{4 r_{F D} I_{0}}{\left(1-r_{F D}+\sqrt{1+r_{F D}^{2}}\right)^{2}}\left(\frac{\tilde{\beta}_{x, y}^{*}}{\beta_{0}^{*}}\right)+\mathcal{O}\left(\frac{\tilde{\beta}_{x, y}^{*}}{\beta_{0}^{*}}\right)^{3}$.

This chromaticity is not only bounded, but in fact even tends to zero at low $\tilde{\beta}^{*}$ thanks to the phasing properties of the presqueezed optics, and can be easily compensated by the other sextupoles of the lattice (see also Sec. II B 1 for the presqueezed optics).

\section{APPENDIX B: SPURIOUS DISPERSION CORRECTION}

Similarly to Eq. (9) which links the off-momentum $\beta$ and $\alpha$ beating to the $(2,0)$ and $(0,2)$ chromatic driving terms, the spurious dispersion and angular dispersion induced by a nonzero closed orbit are directly connected to the following quantities:

$$
\begin{aligned}
& {\left[\frac{\Delta D_{x, y}}{\sqrt{\beta_{x, y}}}+j\left(\sqrt{\beta_{x, y}} \Delta D_{x, y}^{\prime}+\alpha_{x, y} \frac{\Delta D_{x, y}}{\sqrt{\beta_{x, y}}}\right)\right]_{s=s_{0}}} \\
& \quad \propto c_{(1,0),(0,1)}\left(s_{0}\right) \\
& \stackrel{\text { def }}{=} \int_{s_{0}}^{s_{0}+C} d s\left[K_{1}(s)-K_{2}(s) D_{x}(s)\right] \\
& \quad \times \sqrt{\beta_{x, y}(s)} \Delta z_{x, y}(s) e^{j\left[\mu_{x, y}(s)-\mu_{x, y}\left(s_{0}\right)\right],}
\end{aligned}
$$

where $\Delta z_{x, y}(s)$ denotes the horizontal and vertical closed orbits around the machine.

The contribution of the inner triplet is rather easy to establish. Indeed, when the optics is squeezed, the betatron phase advances between the IP and the final focus quadrupoles is very close to $\pi / 2$. As a result, the evolution of the beam orbit in the left and right inner triplets is simply given by

$$
\Delta z^{L, R}(s)=\mp \Theta_{c} \sqrt{\beta(s) \beta^{*}}=\mp \frac{d_{\mathrm{bb}}}{2} \sqrt{\epsilon \beta(s)},
$$

where the suffixes $x$ and $y$ have been omitted for the sake of clarity, $d_{\mathrm{bb}}$ represents the normalized beam-beam separation introduced in Eq. (37), $\epsilon$ is the physical transverse emittance of the beam, the $\mp$ sign stands for the left and right sides of the IP, respectively, when the crossing angle is positive, and conversely when it is negative. Under these conditions, the normalized kick of angular dispersion induced by each inner triplet is directly connected to the integral $I_{x, y}^{L, R}$ previously introduced in Eq. (3):

$$
\begin{aligned}
\sqrt{\frac{\beta}{\epsilon}} \Delta D^{\prime} & =\int_{\text {triplet }(L, R)} d s K_{1}(s) \sqrt{\beta(s) / \epsilon} \Delta z^{L, R}(s) \\
& = \pm I^{L, R} \times \frac{d_{\mathrm{bb}}}{2},
\end{aligned}
$$

where the \pm sign depends on the sign of the crossing angle, on the transverse plane ( $\mathrm{H}$ or $\mathrm{V})$, and on the triplet ( $\mathrm{L}$ or $\mathrm{R}$ ) under consideration, but does not need to be specified further.

Concerning the contribution of the orbit bumps in the arcs, it is first important to notice that the quantity $K_{1}(s)-$ $K_{2}(s) D_{x}(s)$ [see the rhs of Eq. (B1)] is marginal in the main quadrupoles which are equipped with a sextupole belonging to one of the weak families. This comes from the fact 
that the chromaticity induced by each of these quadrupoles is corrected very locally by the weak sextupole which is directly attached to it, and keeping in mind that the weak sextupole families are operated at strictly constant normalized strength as of the injection optics until the end of the telescopic squeeze (see Sec. II B 1). For the other arc quadrupoles, equipped with a strong sextupole, this quantity is reduced to $-\Delta K_{2}(s) D_{x}(s)$ where, as in Sec. II B 1 , $\Delta K_{2}(s)$ denotes the additional strength which is given to the strong sextupoles for the chromatic correction of the inner triplet. In a given arc and a given plane, the orbit bump $\Delta z_{\mathrm{co}}(s)$ is then achieved by acting on two orbit correctors upstream of the first strong sextupole of that arc, and closed downstream of the last one before reaching the IP. This offers the possibility to adjust both the normalized amplitude, namely $d_{\mathrm{co}}$, and the phase $\phi_{\mathrm{co}}$ of the bump with respect to the sextupole families participating to the chromatic correction of the triplet:

$$
\Delta z_{\mathrm{co}}(s)=d_{\mathrm{co}} \sqrt{\epsilon \beta(s)} \cos \left[\phi_{\mathrm{co}}+\mu(s)\right] .
$$

Combining Eqs. (B1), (B3), and (B4), the conditions to be satisfied for the correction of the spurious dispersion are then very similar to the ones established in Eq. (12) for the correction of the off-momentum $\beta$ beating. Using the same notations, one gets

$$
\begin{aligned}
& d_{x_{\mathrm{co}}}^{L, R} e^{j\left(\Delta \mu_{x}^{L, R}-\pi / 2\right)} \times N_{F}^{L, R} \beta_{x_{F}} D_{x_{F}}\left[\cos \left(\phi_{x_{\mathrm{co}}}^{L, R}\right) \Delta K_{2_{F}}^{L, R}\right. \\
& \left.\quad+e^{j \Delta \mu_{x_{F D}}^{L, R}} N_{D}^{L, R} \beta_{x_{D}} D_{x_{D}} \cos \left(\phi_{x_{\mathrm{co}}}^{L, R}+\Delta \mu_{x_{F D}}^{L, R}\right) \Delta K_{2_{D}}^{L, R}\right] \\
& \equiv \\
& d_{x_{\mathrm{bb}}} I_{x}^{L, R}, \\
& d_{y_{\mathrm{co}}}^{L, R} e^{j\left(\Delta \mu_{y}^{L, R}-\pi / 2\right)} \times\left[e^{-j \Delta \mu_{y_{F}}^{L, R}} N_{F}^{L, R} \beta_{y_{F}} D_{x_{F}}\right. \\
& \quad \times \cos \left(\phi_{y_{\mathrm{co}}}^{L, R}-\Delta \mu_{y_{F D}}^{L, R}\right) \Delta K_{2_{F}}^{L, R} \\
& \left.\quad+N_{D}^{L, R} \beta_{y_{D}} D_{x_{D}} \cos \left(\phi_{y_{\mathrm{co}}}^{L, R}\right) \Delta K_{2_{D}}^{L, R}\right] \equiv-\frac{d_{y_{\mathrm{bb}}}}{2} I_{y}^{L, R},
\end{aligned}
$$

where the \pm sign which was left unspecified in Eq. (B3) has been implicitly absorbed in the definition of the phases $\phi_{x_{\mathrm{co}}, y_{\mathrm{co}}}^{L, R}$ of the orbit bumps (i.e. by eventually shifting them by $\pi$ ).

First of all, assuming that the presqueezed optics is set up with a crossing angle which is already dimensioned for the collision optics (i.e. overdimensioned by the factor $\sqrt{\beta_{\text {presqueezed }}^{*} / \beta_{\text {squeezed }}^{*}}$, the telescopic squeeze can be achieved at constant crossing angle. Under this condition, if the correction of the spurious dispersion is set up for the presqueezed optics, it will still be valid all along provided a (slight) reduction of the bump amplitude by the scaling factor $\lambda$ introduced in Eq. (24). Indeed, the dispersion can be seen as a dispersive orbit which, if corrected locally, is strictly insensitive to the optics manipulations which are performed by the left and right supporting insertions during the telescopic part of the squeeze. The same observation stands for the crossing bumps and orbit bumps in the arcs if the latter do not overlap with the supporting insertions. On the other hand, any potential dispersion or orbit leakage due to machine imperfections will of course be further amplified during the telescopic squeeze. Finally, since the strength of the strong sextupoles shall be (slightly) increased by the $\lambda$ factor during the telescopic squeeze (see Sec. II B 2), the bump amplitude in the arcs shall even be reduced accordingly during this process.

It is then sufficient to inspect the solution of Eq. (B5) for the presqueezed optics only, for which the $\beta$ functions $\beta_{x_{F, D}, y_{F, D}}$ at the strong sextupoles SF and SD are linked by the relations (13), the betatron phase advances $\Delta \mu_{x_{F D}, y_{F D}}^{L, R}$ between two consecutive strong SF and SD are discussed in Eq. (14), the settings of the strong sextupoles have been established in Eq. (19), and the left and right IR phase advances $\Delta \mu_{x, y}^{L, R}$ are defined in Eq. (22). By replacing each occurrence of $\cos (\phi)$ in Eq. (B5) by its equivalent expression $\left[e^{j \phi}+e^{-j \phi}\right] / 2$, and since the lhs of the achromaticity conditions (12) is real by construction of the presqueezed optics, one can immediately connect the left and right phase advances $\Delta \mu_{x, y}^{L, R}$ of the high luminosity insertions to the phases $\phi_{x_{\mathrm{co}}, y_{\mathrm{co}}}^{L, R}$ wich are appropriate for the orbit bumps in the arcs:

$$
\phi_{x_{\mathrm{co}}, y_{\mathrm{co}}}^{L, R} \equiv \Delta \mu_{x, y}^{L, R}-\frac{\pi}{2} \bmod [\pi] \sim \pm \xi^{L, R} \frac{r_{F D}}{2} \bmod [\pi],
$$

where the aspect ratio $r_{F D}$ is defined in Eq. (17), and the quantities $\xi^{L, R}= \pm 1$ depend on the choice of the strong sextupole families [see Eq. (14)]. Inserting (B6) into (B5), and using the sextupole settings given in Eq. (19), the normalized amplitude of the bumps then follows immediately for the presqueezed optics:

$$
d_{x_{\mathrm{co}}, y_{\mathrm{co}}}^{L, R}=\underbrace{\frac{1}{1-\kappa}}_{\substack{\stackrel{\text { def }}{=} \lambda_{0}}}\left[1 \pm\left|\frac{\Delta I_{0}}{I_{0}}\right|\right] \times \frac{d_{\mathrm{bb}}}{2},
$$

where the $\kappa$ constant was already introduced in Eq. (26), the average triplet-related quantities $I_{0}$ and $\Delta I_{0}$ are the same in the two planes for a round presqueezed optics [see Eq. (6), with $\left|\Delta I_{0} / I_{0}\right| \sim 10-20 \%$ for typical (HL)-LHC triplets], and the \pm sign shall flip from the left to the right of the IP in a given plane, and between the $\mathrm{H}$ and $\mathrm{V}$ planes on a given side of the IP. For instance, a rapid inspection of Fig. 11 shows that for the LHC triplets in IR1 and IR5 (which are of same polarity), and the clockwise rotating Beam1, this sign shall be positive both on the left side of the IP for horizontal crossing (IP5), and on the right side of the IP for vertical crossing (IP1), and negative in the other two cases. The opposite rules would stand for the counterclockwise rotating Beam2.

As mentioned previously, the amplitude of the bumps shall then be reduced during the telescopic squeeze, in 
proportion with the increase of strength of the strong sextupoles by the scaling factor $\lambda$ defined in Eq. (25). Surprisingly enough, this factor $\lambda$ converges also quite rapidly at low $\beta^{*}$ towards the constant $\lambda_{0}$ defined above in Eq. (B7). Consequently, for telescopic optics of sufficiently low $\beta^{*}$, and neglecting the left-right imbalance $\left|\Delta I_{0} / I_{0}\right|$, the normalized amplitude of the bumps is given by the very simple following expression:

$$
d_{\mathrm{co}} \sim \frac{d_{\mathrm{bb}}}{2}
$$

which varies very smoothly with the choice of gradient of the final focus quadrupoles, only via the minimum normalized beam-beam separation needed, which shall be slightly increased for weaker and longer quadrupoles of larger aperture.

[1] CERN Report No. CERN-2004-003-V-1, 2004.

[2] L. Rossi, in Proceedings of the 2nd International Particle Accelerator Conference, San Sebastiàn, Spain, 2011 (EPS-AG, Spain, 2011), p. 908.

[3] S. Fartoukh, CERN Report No. CERN-LHC-ProjectReport-308, 1999.

[4] J.-P. Koutchouk, in Proceedings of the 18th Biennial Particle Accelerator Conference, New York, NY, 1999 (IEEE, New York, 1999), p. 372.

[5] J.P. Koutchouk, L. Rossi, and E. Todesco, CERN Report No. CERN-LHC-Project-Report-1000, 2007.

[6] O. Brüning, R. De Maria, and R. Ostojic, CERN Report No. CERN-LHC-Project-Report-1008, 2007.

[7] S. Fartoukh, CERN Report No. CERN-ATS-2010-026, 2010, pp. 262-290.

[8] S. Fartoukh, CERN Report No. sLHC-Project-Report0049, 2010.

[9] M. Korostelev, E. Cruz-Alaniz, D. Newton, A. Wolski, O. Brüning, and R. Tomás, in Proceedings of the 4th International Particle Accelerator Conference 2013, Shanghai, China, MOPWO063.

[10] D. Trbojevic (private communication).

[11] http://mad.web.cern.ch/mad.

[12] R. Ostojic, CERN Report No. CERN-ATS-2010-026, 2010, pp. 253-257.

[13] R. De Maria and S. Fartoukh, CERN Report No. sLHCProject-Report-0055, 2011.

[14] R. Calaga, R. Tomas, and F. Zimmermann, in Proceedings of the 1st International Particle Accelerator Conference 2010, Kyoto, Japan, 2010 (ICR, Kyoto, 2010), p. 1240.

[15] S. Fartoukh and O. Brüning, CERN Report No. LHCProject-Report 501, 2001.

[16] E. Todesco, CERN Report No. CERN-AB 2005-014 ADM, pp. 228-234.

[17] E. Todesco, B. Bellesia, L. Bottura, A. Devred, V. Remondino, S. Pauletta, S. Sanfilippo, W. Scandale, C.
Vollinger, and E. Wildner, in Proceedings of the MT-18 Conference [IEEE Trans. Appl. Supercond. 14, 177 (2004)].

[18] S. Fartoukh and M. Giovannozzi, Nucl. Instrum. Methods Phys. Res., Sect. A 671, 10 (2012).

[19] R. De Maria and S. Fartoukh, CERN Report No. sLHCProject-Report-0050, 2010.

[20] Y. Nosochkov, Y. Cai, M-H. Wang, S. Fartoukh, M. Giovannozzi, R. de Maria, and E. McIntosh, in Proceedings of the 4th International Particle Accelerator Conference 2013, Shanghai, China, TUPFI016.

[21] R. De Maria, S. Fartoukh, M. Giovannozzi, A. Chanće, B. Dalena, J. Payet, J. Resta-Ĺopez, K. M. Hock, M. Korostelev, and A. Wolski, in Proceedings of the 4th International Particle Accelerator Conference 2013, Shanghai, China, WEPEA047.

[22] S. Fartoukh, CERN Report No. CERN-ATS-2012-069, pp. 128-134.

[23] S. Fartoukh and R. De Maria, in Proceedings of the 3rd International Particle Accelerator Conference, New Orleans, LA, 2012 (IEEE, Piscataway, NJ, 2012), p. 145.

[24] R. De Maria, S. Fartoukh, A. Bogomyakov, and M. Korostolev, in Proceedings of the 4th International Particle Accelerator Conference 2013, Shanghai, China, TUPFI014.

[25] S. Fartoukh et al., CERN Report No. CERN-ATS-Note2011-033 MD, 2011.

[26] S. Fartoukh et al., CERNReport No. CERN-ATS-Note2011-060 MD, 2011.

[27] S. Fartoukh, R. Tomas, B. Goddard, W. Hofle, D. Jacquet, G. Kruk, M. Lamont, R. de Maria, R. Miyamoto, G. Mueller, M. Pojer, L. Ponce, S. Redaelli, N. Ryckx, R. Steinhagen, M. Strzelczyk, G. Vanbavinckhove, and J. Wenninger, CERN Report No. CERN-ATS-Note-2011132 MD, 2011.

[28] S. Fartoukh, V. Kain, Y. Levinsen, E. Maclean, R. de Maria, T. Person, M. Pojer, L. Ponce, S. Redaelli, P. Skowronski, M. Solfaroli, R. Tomas, and J. Wenninger, CERN Report No. CERN-ATS-Note-2013-004 MD, 2013.

[29] T. Risselada (private communication).

[30] R. Bruce, R. W. Assmann, L. Lari, and S. Redaelli, in Proceedings of the LHC Machine Protection Workshop, Annecy, France, 2013 (unpublished).

[31] A. Bogomyagkov and R. De Maria, in Proceedings of the 4th International Particle Accelerator Conference 2013, Shanghai, China, TUPFI015.

[32] R. Tomas, T. Bach, R. Calaga, A. Langner, Y. I. Levinsen, E. H. Maclean, T.H. B. Persson, P. K. Skowronski, M. Strzelczyk, G. Vanbavinckhove, and R. Miyamoto, Phys. Rev. ST Accel. Beams 15, 091001 (2012).

[33] Brookhaven National Laboratory Report No. BNL-51932, 1986.

[34] W. Herr, Reports No. CERN SL/93-45 (AP) and LHCNote-258, 1993.

[35] L. Leunissen, CERN Report No. LHC-Project-Report298, 1999.

[36] http://hilumilhc.web.cern.ch. 